\title{
A linear numerical model for analysing the hydroelastic response of a flexible Electroactive Wave Energy Converter
}

\author{
Aurélien Babarit ${ }^{\mathrm{a}}$, Jitendra Singh ${ }^{\mathrm{a}}$, Cécile Mélis ${ }^{\mathrm{b}}$, Ambroise Wattez ${ }^{\mathrm{b}}$, \\ Philippe Jean ${ }^{\mathrm{b}}$ \\ ${ }^{a}$ Ecole Centrale de Nantes - CNRS, 1 rue de la Noe, 44300 Nantes, France \\ ${ }^{b}$ SBM Offshore, 24 avenue de Fontvieille, Monaco MC98007
}

\begin{abstract}
In this paper, a linear mathematical and numerical model for analysing the dynamic response of a flexible electroactive wave energy converter is described. The Wave Energy Converter (WEC) is a floating elastic tube filled with slightly pressurised sea water. It is made of Electroactive Polymers (EAPs). Under simplifying assumptions, a set of governing equations is formulated for the flow inside the tube, the flow outside the tube and the behaviour of the tube wall. By combining them, the evolution of the flow velocity in the tube can be written as a wave equation. The corresponding eigenmodes of vibration are calculated. Then, using spectral decomposition, the equation of motion for the response of the tube in waves is derived. Experiments were carried out on a scale model of the wave energy converter in the wave tank of Ecole Centrale de Nantes in 2011. Numerical results are
\end{abstract}

Email addresses: aurelien.babarit@ec-nantes.fr (Aurélien Babarit), vishist.jiten@gmail.com (Jitendra Singh), cecile.melis@sbmoffshore.com (Cécile Mélis), ambroise.wattez@sbmoff shore.com (Ambroise Wattez), philippe.jean@sbmoff shore.com (Philippe Jean)

Now with IBM 
compared with experimental results in regular waves, showing rather good agreement, which validates the model and the initial modelling assumptions. Finally, estimates are made for the energy performance of a possible prototype.

Keywords: Wave energy converter, Numerical modelling, Flexible tube, Electroactive Polymer

\section{Introduction}

Over the four last decades, several hundred projects for ocean wave energy conversion have been initiated globally. A useful classification and explanation of the different working principles of Wave Energy Converters (WECs) can be found in [6]. In most cases, wave energy converters rely on one or several interconnected rigid bodies which serve as the primary wave absorbers. Since these bodies are rigid, they experience very large forces and stress concentrations in extreme seas. As a result, their structures and mooring systems are expensive, and hence also the cost of the energy they yield.

Recently, flexible or deformable WECs have been proposed, such as the Anaconda WEC [10] or the SBM S3 WEC[16]. These devices consist of water-filled horizontal elastic tubes floating just beneath the sea surface. They are expected to have to cope with significantly smaller structural loads and mooring forces in extreme conditions in contrast with rigid WECs. Thus, it is expected that the cost of energy will be significantly smaller for these devices.

When ocean waves travel above a device like the Anaconda WEC or the SBM S3 WEC, see figure (1), they apply a time-varying pressure on 
the tube wall which induces local changes in diameter. This effect creates bulge waves in the tube. The work done by the pressure differential applied on the radial moving tube surface is the energy harvested from the ocean waves. A major problem is how to convert the harvested power to useful output power. For the Anaconda WEC, it was proposed to use power takeoff (PTO) systems composed of hydraulic valves, hydraulic accumulators and turbines. The power take-off can be located at the stern of the tube or distributed along the tube [9]. In the SBM S3 WEC, the PTO consists of rings of electroactive polymers (EAP) distributed along the tube [13]. Energy harvesting is achieved through the deformation of the tube, thanks to the electroactive properties of the EAP rings. One can note that the use of electroactive material in the context of wave energy conversion had previously been proposed by [5], but with a different implementation.

Theoretical one-dimensional models for the dynamic response of the Anaconda WEC have been proposed in [9] and in [4]. In [9], the theoretical model predicts that the bulge wave amplitude grows linearly along the tube. Comparisons of numerical predictions with experimental results show fair agreement provided that losses in the tube wall and in the radiated wave field are taken into account. Distensibility and losses were calibrated against the experiments. In [4], the proposed model is more comprehensive. It takes into account the boundary conditions and the power take-off. At the bow, the condition is a no-flow condition. At the stern, the condition is a relationship between the flow and the pressure which depends on the impedance of the power take-off system. A general solution for bulge wave propagation is given which includes forward and backward propagative terms (whereas 
there is only a forward propagative term in the solution of [9]). Experiments with a flexible tube are described. Comparisons of theoretical and experimental results show good agreement for absorbed power performance (after calibration of the losses). For wave components, despite strong similarities between experimental and predicted data, quantitative agreement is not very good.

In the present study, the focus is on the SBM S3 WEC. For this particular wave energy converter, the models that were derived for the Anaconda WEC are not suitable for several reasons. Firstly, the boundary conditions are different. Indeed, the condition at the stern is a no-flow condition in case of the SBM S3 WEC. The tube is also allowed to move horizontally whereas the models developed for the Anaconda WEC assumes that the device is fixed. Secondly, the longitudinal tension is not taken into account in the models of the Anaconda WEC. In [4], it is neglected because it was found that it has a small effect on the distensibility. For the SBM S3 WEC, we found that it is critical for matching the boundary conditions for the tube section (no deformations at both ends of the tube) and for achieving good agreement between experimental and predicted eigenperiods. Thirdly, the models for the Anaconda WEC require calibration of the distensibility and losses using experimental data. Thus the model is not suitable for prediction of the power performance of a full scale device. Finally, quantitative agreement for the wave components is not very good in [4]. This is an issue for the SBM S3 WEC because power absorption occurs at the EAP rings which are distributed along the tubes. Errors on the amplitudes of the wave components would transfer directly to errors of the same order of magnitude 
in power absorption performance.

Therefore, to assess and optimize the performance of the SBM S3 device, one needs a model that rectifies the deficiencies of the models of [9] or [4], which is the primary aim of this study. The proposed mathematical and numerical model is described in sections 2 and 3. The model is validated against experimental results in section 5 which are described in section 4 . Using the numerical model, preliminary estimates of power absorption potential are made and provided in section 6 .

\section{Governing equations}

In this section, governing equations are derived for all the aspects that need to be taken into account in the numerical model of the device. Thus, sub-models are derived for describing the behaviour of the fluid inside the tube (inner flow problem), outside the tube (outer flow problem) and the motion of the tube and its wall (structural problem).

The modelling problem central to this study has clear similarities with the modelling of blood flow in arteries [11]. However, there are significant differences, namely the coupling with the outer flow, the boundary conditions (in this study the tube is closed at both ends whereas there are flow conditions for blood flow in arteries) and the considerably greater time and length scales.

\subsection{Notations and assumptions}

A tube of length $L$ made up of an incompressible elastic material is considered. At rest, let $r_{S}$ denote the radius, $z_{S}$ the submergence of the horizontal axis of the tube, $\rho_{\text {tube }}$ the density of the tube and $h_{S}$ its wall thickness. The thickness is assumed to be small with respect to the diameter. The tube 


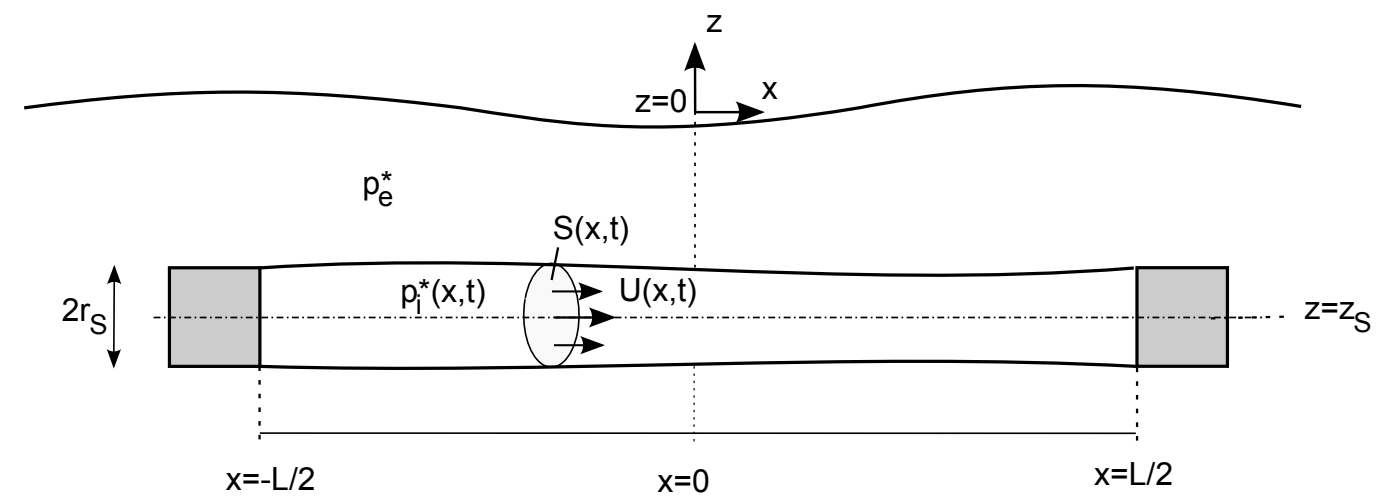

Figure 1: Submerged elastic tube in waves

mass is $M_{\text {tube }}=2 \rho_{\text {tube }} \pi r_{S} h_{S} L$. The area of a section of the tube of horizontal coordinate $x$ at time $t$ is denoted by $S(x, t)$.

By construction, the tube wall is reinforced in the longitudinal direction with rigid fibres so that the tube cannot deform in the $\mathrm{x}$-direction. The static pressure inside the tube is higher than the external pressure, so that the elastomeric wall has a static pre-strain. Hence, the motion of the tube in the $\mathrm{x}$-direction, denoted here by $X$, is a rigid body motion (usually referred to as surge motion in naval architecture). It is assumed that ocean waves propagate only in the $\mathrm{x}$-direction. Thus, there is no lateral movement for symmetry reasons. Furthermore, vertical deformations are not taken into account in this analysis for the sake of simplicity. Indeed, further work (not reported here) showed that vertical and radial deformations are coupled at first order only through hydrodynamic coefficients that are small with respect to the other physical effects (see section 5.1 for the hydrodynamic coefficients for the radial deformations).

At each end, the tube is embedded in a towhead of mass $M_{\text {towhead }}$. Each 
towhead is connected to the sea bottom by means of a slack mooring system.

\subsection{Inner flow}

For the inner flow, a one-dimensional model is used. Such models are commonly used for modelling blood flow in arteries [11].

Following [17] (page 71), it is assumed:

- Axial symmetry Let $(R, \theta, x)$ be the cylindrical coordinates. It is assumed that all quantities are independent of the angular coordinate $\theta$. Thus, the bulge (deformation) of the section $S$ remains circular and depends only on the horizontal coordinate $x$ and on time $t$.

- Radial displacement The tube wall moves only in the radial direction. The radius $r$ is a function of the time $t$ and the horizontal coordinate $x, r=r(x, t)$.

- Constant pressure Let $p_{i}^{*}=p_{i}-\rho g z+p_{i S}$ be the total pressure in the tube, where $p_{i S}$ and $p_{i}$ are the static and dynamic pressures, respectively. At equilibrium, the dynamic pressure $p_{i}=0$. Moreover, it is assumed to be constant on each axial section: $p_{i}=p_{i}(x, t)$.

- Dominance of axial velocity Let $(u, v, w)$ be the three components of the fluid velocity in the $\mathrm{x}, \mathrm{y}$ and $\mathrm{z}$ directions, respectively. The fluid velocities in the directions perpendicular to the axis of the tube are assumed to be small with respect to $u$, i.e. $v \approx w \ll u$. Let us define $Q(x, t)=\int_{S} u d S$ to be the volume flux through section $S(x)$ and $U=\frac{Q}{S}$ the section averaged flow velocity. It is assumed that the flow velocity 
$u$ can be described by the product of the section averaged flow velocity $U$ with the velocity profile $\psi\left(\frac{R}{r(x, t)}\right)$ :

$$
u(R, x, t)=U(x, t) \psi\left(\frac{R}{r(x, t)}\right)
$$

Under these assumptions, the equations governing the flow inside the tube are:

- Mass conservation

$$
\frac{\partial S}{\partial t}=-\frac{\partial Q}{\partial x}=-\frac{\partial}{\partial x}(S U)
$$

- Momentum conservation:

$$
\frac{\partial}{\partial t}(S U)+\frac{\partial}{\partial x}\left(\varsigma S U^{2}\right)=-\frac{S}{\rho} \frac{\partial p_{i}^{*}}{\partial x}-B_{R} U
$$

where $B_{R}$ is a damping parameter related to viscosity and $\varsigma$ is the momentum correction coefficient defined by:

$$
\varsigma=\frac{\int_{S} u^{2} d x}{S U^{2}}=\frac{\int_{S} \psi^{2} d x}{S}
$$

Following [17] (page 75), the viscous damping parameter is assumed to be equal to $B_{R}=2 \pi \nu \psi^{\prime}(1)$ where $\nu$ is the kinematic viscosity.

In the following, it is further assumed that all flow-related physical quantities exhibit only small variation from their equilibrium value. Therefore, all second order effects are disregarded. Equations (2) and (3) simplify to:

$$
\frac{\partial S}{\partial t}=-S_{S} \frac{\partial U}{\partial x}
$$




$$
\frac{\partial U}{\partial t}=-\frac{1}{\rho} \frac{\partial p_{i}^{*}}{\partial x}-\frac{B_{R}}{S_{S}} U=-\frac{1}{\rho} \frac{\partial p_{i}}{\partial x}-\frac{B_{R}}{S_{S}} U
$$

where $S_{S}\left(=\pi r_{S}^{2}\right)$ is the area of the tube at static equilibrium.

This model for the inner flow is similar to the one of [9] except that it takes into account viscous damping due to wall friction.

\subsection{Outer flow}

Linear potential flow theory is used for the modelling of the flow outside the tube. It is based on the following assumptions:

- The fluid is inviscid and the flow is irrotational. Therefore, there exists a velocity potential $\Phi$ from which the velocity can be derived in the outer fluid domain $\Omega$.

- The flow is incompressible. Using mass conservation, this assumption leads to Laplace's equation: $\Delta \Phi=0$ everywhere in the fluid domain.

- The ratios of wave height to wavelength (i.e wave steepness) must be small.

- The motion of the tube and the deformations are small and around a fixed mean position. That is, the ratio of the typical amplitude of horizontal motion to the length of the tube and the ratio of the amplitude of deformation to the radius of the tube are small.

As a consequence of the linearity hypothesis, the different components of the outer flow can be treated separately as diffraction and radiation problems for which the diffracted potential $\Phi_{D}$ and radiation potential $\Phi_{R}$ are solutions of the following boundary value problems: 
- Diffraction problem

$$
\left\{\begin{array}{cc}
\Delta \Phi_{D}=0 & \text { in } \Omega \\
\frac{\partial \Phi_{D}}{\partial n}=-\frac{\partial \Phi_{I}}{\partial n} & \text { at sea bottom } S_{\text {bottom }} \\
\frac{\partial^{2} \Phi_{D}}{\partial t^{2}}+g \frac{\partial \Phi_{D}}{\partial z}=0 & \text { at free surface } S_{F S} \\
\frac{\partial \Phi_{D}}{\partial n}=-\vec{\nabla} \Phi_{I} \cdot \vec{n} & \text { on wet body surface } S_{B}
\end{array}\right.
$$

- Radiation problem

$$
\left\{\begin{array}{cc}
\Delta \Phi_{R}=0 & \text { in } \Omega \\
\frac{\partial \Phi_{R}}{\partial n}=0 & \text { at sea bottom } S_{b o t t o m} \\
\frac{\partial^{2} \Phi_{R}}{\partial t^{2}}+g \frac{\partial \Phi_{R}}{\partial z}=0 & \text { at free surface } S_{F S} \\
\frac{\partial \Phi_{R}}{\partial n}=\vec{V} \cdot \vec{n} & \text { on wetted body surface } S_{B}
\end{array}\right.
$$

In the above boundary value problems $\vec{n}$ is the normal vector to the body surface oriented towards the outer fluid, $\Phi_{I}$ is the incident wave potential and $\vec{V}$ is the body velocity. The total velocity potential $\Phi$ is the sum of the incident, diffraction and radiation potential $\Phi=\Phi_{I}+\Phi_{D}+\Phi_{R}$.

The diffraction and radiation boundary value problems can be solved efficiently in the frequency domain using Boundary Element Method (BEM) based numerical tools such as WAMIT [14] or NEMOH [2].

Once the velocity potential has been obtained, the dynamic component of the outer pressure field $p_{e}$ can be written as the sum of an excitation pressure $p_{e x}=-\rho\left(\frac{\partial \Phi_{I}}{\partial t}+\frac{\partial \Phi_{D}}{\partial t}\right)$, a function of the incident and diffracted wave fields, and a radiation pressure $p_{\text {rad }}=-\rho \frac{\partial \Phi_{R}}{\partial t}$, a function of the surge and the bulge motions of the tube. The total outer pressure will be obtained by adding the contribution of the hydrostatic pressure. Eventually, the outer pressure $p_{e}^{*}$ is 
given by:

$$
p_{e}^{*}=\overbrace{p_{e x}+p_{\text {rad }}}^{p_{e}}-\rho g z
$$

\subsection{Linearised wall equation}

For sake of clarity, only the main steps leading to the derivation of the linearised wall equation are given in this section. The details of the derivation can be found in Appendix A.

The linearised wall equation is obtained by first considering a small piece of the tube wall. It is assumed that (i) the tube mass is so small that the inertia effects can be neglected, (ii) that the deformations are small, (iii) that the tube is thin, i.e its thickness $h$ is small with respect to the radius $r(h \ll r)$ and (iv) that the tube at rest is a straight horizontal cylinder. Using those four assumptions, one can write a non-linear relationship (equation (A.6)) between the internal dynamic pressure $p_{i}$, the contour-averaged external pressure $\bar{p}_{e}=\int_{C(x)} p_{e}(M) d C$ (where $C(x)$ is the circular contour of the section area $S(x)$ ), the longitudinal static pretension in the reinforcing fibres $T_{S}$, the section area $S$ and the hoop stress in the wall $\sigma_{\theta}$.

To close the problem, the hoop stress $\sigma_{\theta}$ must be related to the deformations. Dedicated experiments were carried out (see Appendix A). It was found that the tube material behaves non-linearly. A visco-hyperelastic model has been used. By linearising around static equilibrium, one can relate the hoop stress $\sigma_{\theta}$, the section area $S$ and the time derivative of the section area $\dot{S}$ (equation (A.9)). Eventually, by linearising this last equation and combining with equation (A.9), one obtains the linear wall equation: 


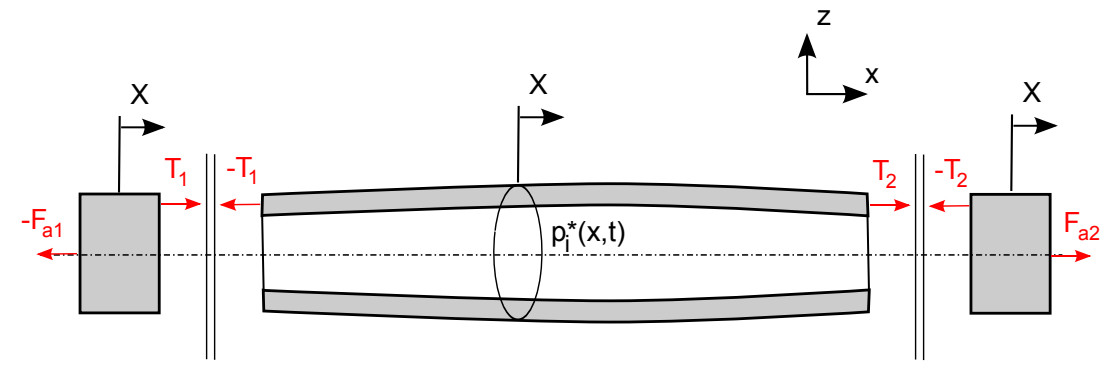

Figure 2: The three body problem for determination of the equation of motion in surge.

$$
p_{i}=\bar{p}_{e}-\frac{1}{D S_{S}} \frac{1}{K_{0}^{2}} \frac{\partial^{2} S}{\partial x^{2}}+\frac{1}{D S_{S}}\left(S-S_{S}\right)+\frac{\rho}{S_{S}} \eta \dot{S}
$$

where $D$ is the distensibility [9], $\frac{1}{K_{0}^{2}}=\frac{T_{S} D}{4 \pi}$ and $\eta$ is the material damping coefficient. Note that the equation is similar to the one used in [9] except that it takes into account the effect of longitudinal tension $\left(-\frac{1}{D S_{S}} \frac{1}{K_{0}^{2}} \frac{\partial^{2} S}{\partial x^{2}}\right.$ term in equation 10).

It must be noted that the distensibility $D$ and the material damping coefficients were characterized through dedicated experiments. This lead to significant uncertainties on the values of these coefficients, see Appendix A. The uncertainty on the distensibility coefficient is $[-20 \%,+10 \%]$.

\subsection{Horizontal motion}

In contrast to the models of [9] and [4], the tube is allowed to move horizontally. As previously mentioned, the tube is reinforced with inextensible fibres so that it can not deform in the horizontal direction. Therefore, the surge motion is a rigid body motion. To determine its governing equation, the tube is split into three parts: the elastic (central) part and the two towheads at the extremities, as shown in figure 2. By applying Newton's law for 
the left towhead and the rigth towhead, one can write:

$$
\begin{aligned}
M_{\text {towhead }} \ddot{X} & =F_{a 1}+T_{1}-\left.p_{i}^{*}\right|_{-\frac{L}{2}} S_{S}+\left.p_{e}^{*}\right|_{-\frac{L}{2}} S_{S} \\
M_{\text {towhead }} \ddot{X} & =F_{a 2}-T_{2}+\left.p_{i}^{*}\right|_{\frac{L}{2}} S_{S}-\left.p_{e}^{*}\right|_{\frac{L}{2}} S_{S}
\end{aligned}
$$

where:

- $F_{a 1}$ and $F_{a 2}$ are the mooring forces on the left and right towheads. In this study, they are both modelled as linear springs:

$$
\begin{aligned}
& F_{a 1}=-K_{a} X-F_{a S} \\
& F_{a 2}=-K_{a} X+F_{a S}
\end{aligned}
$$

where $K_{a}$ is the mooring stiffness coefficient, and $F_{a S}$ the static mooring pretension.

- $T_{1}$ and $T_{2}$ are the tensions in the tube wall at the left and right ends, respectively.

For the central part, by applying Newton's law to a short section of the tube, by retaining only first order terms, and by using equation (A.3), one can show:

$$
2 \pi \rho_{\text {tube }} r_{S} h_{S} \ddot{X}=\frac{\partial T}{\partial x}-h_{S} \sigma_{\theta S} \frac{\partial r}{\partial x}
$$

where $\sigma_{\theta S}$ is the hoop stress at static equilibrium.

By integrating over the length of the tube and by using the fact that the two extremities of the tube are embedded in towheads (and thus cannot deform: $\left.r\left( \pm \frac{L}{2}\right)=r_{S}\right)$, one can show:

$$
M_{\text {tube }} \ddot{X}=T_{2}-T_{1}
$$


Then, first, let us consider equation (11) and eqution (12) at static equilibrium. One can show:

$$
T_{S}=F_{a S}+p_{i S} S_{S}
$$

Then, by adding equations (11), equation (12) and equation (16) the governing equation for the surge motion is obtained:

$$
M \ddot{X}=-2 K_{a} X+\left(\Delta p_{i}-\Delta p_{e}\right) S_{S}
$$

where $M=M_{\text {tube }}+2 M_{\text {towhead }}, \Delta p_{i}=\left.p_{i}\right|_{\frac{L}{2}}-\left.p_{i}\right|_{-\frac{L}{2}}$ and $\Delta p_{e}=\left.p_{e}\right|_{\frac{L}{2}}-\left.p_{e}\right|_{-\frac{L}{2}}$. This equation shows that the surge motion is coupled with the internal flow through the pressure difference term. Alternatively, equation (18) can be written as:

$$
M \ddot{X}=-2 K_{a} X-\rho S_{S} \int_{-\frac{L}{2}}^{\frac{L}{2}} \frac{\partial U}{\partial t} d x-\Delta p_{e} S_{S}
$$

by integrating equation (6) from $-\frac{L}{2}$ to $\frac{L}{2}$.

\subsection{Summary}

In this section, a linear mathematical model for the tube response has been derived. It corresponds to the following system of coupled linear equations:

$$
\left\{\begin{array}{cl}
\frac{\partial S}{\partial t}=-S_{S} \frac{\partial U}{\partial x} & \\
\left.\frac{\partial U}{\partial t}=-\frac{1}{\rho} \frac{\partial p_{i}^{*}}{\partial x}-\frac{B_{R}}{S_{S}} U=-\frac{1}{\rho} \frac{\partial p_{i}}{\partial x}-\frac{B_{R}}{S_{S}} U\right\} & \text { Inner flow model } \\
p_{i}=\bar{p}_{e}-\frac{1}{D S_{S}} \frac{1}{K_{0}^{2}} \frac{\partial^{2} S}{\partial x^{2}}+\frac{1}{D S_{S}}\left(S-S_{S}\right)+\frac{\rho}{S_{S}} \eta \dot{S} & \text { Wall model } \\
p_{e}=-\rho\left(\frac{\partial \Phi_{I}}{\partial t}+\frac{\partial \Phi_{D}}{\partial t}+\frac{\partial \Phi_{R}}{\partial t}\right) & \text { Outer flow model }
\end{array}\right.
$$

complemented with the equation for the horizontal motion of the tube: 


$$
M \ddot{X}=-2 K_{a} X-\rho S_{S} \int_{-\frac{L}{2}}^{\frac{L}{2}} \frac{\partial U}{\partial t} d x-\Delta p_{e} S_{S}
$$

and the two boundary conditions:

$$
U\left( \pm \frac{L}{2}\right)=\dot{X}
$$

and:

$$
S\left( \pm \frac{L}{2}\right)=S_{S}
$$

The first boundary condition follow from the fact that the inner fluid velocity must be equal to the surge velocity at both ends since the ends of the tube are material boundaries. The second boundary condition follow from the fact that the two extremities of the tube are embedded in towheads, and thus cannot deform. Note that in this last equation, it is $S\left( \pm \frac{L}{2}\right)=S_{S}$ rather than $S\left( \pm \frac{L}{2}\right)=S_{0}$ by design of the tube. It is in order to achieve a cylinder with constant section $S_{S}$ all along the tube when inflated at static pressure $p_{i}=p_{i S}$ (and thus $\frac{\partial^{2} r}{\partial x^{2}}=0$ all along the tube).

In equation (20), $\Phi_{R}$ and $\Phi_{D}$ are solutions of the linear boundary value problems $(7)$ and (8). It is recalled that $\Phi_{I}$ is the incident wave potential.

\section{Modal analysis}

\subsection{Wave equation}

Let us define an auxiliary variable $\chi$ such that its time derivative is equal to the inner flow velocity:

$$
\frac{\partial \chi}{\partial t}=U
$$


As a consequence of mass conservation, equation (5), the section area $S$ is related to the spatial derivative of $\chi$ :

$$
S-S_{S}=-S_{S} \frac{\partial \chi}{\partial x}
$$

Then, combining the inner flow equations and the wall equation in equation (20), it follows that $\chi$ is a solution of the wave equation:

$$
\frac{\partial^{2} \chi}{\partial t^{2}}-\frac{1}{\rho D} \frac{\partial^{2} \chi}{\partial x^{2}}+\frac{1}{\rho D} \frac{1}{K_{0}^{2}} \frac{\partial^{4} \chi}{\partial x^{4}}=-\frac{1}{\rho} \frac{\partial \bar{p}_{e}}{\partial x}+\eta \frac{\partial^{2} \dot{\chi}}{\partial x^{2}}+\frac{B_{R}}{S_{S}} \dot{\chi}
$$

\subsection{Eigenmodes}

Let us look for the eigenmodes of response of equation (26). Damping is neglected $\left(\eta=0\right.$ and $\left.B_{R}=0\right)$. The outer fluid is assumed to be at rest, thus the averaged outer pressure $\bar{p}_{\text {ex }}$ is equal to 0 . Radiation effects are also neglected in order to allow analytical derivation of the eigenmodes $\left(\bar{p}_{\text {rad }}=0\right)$. The validity of this last approximation depends on the importance of radiation effects in the dynamic response of the system. In [4], the surrounding water is found to have a small effect for the case of a deeply submerged tube. In our case, due to practical constraints only tubes of small diameters have been considered until now. For small diameters, radiation effects are expected to be small. However for tubes of large diameters, radiation effects may become much more significant and may have to be taken into account. Note that neglecting the radiation effects does not make the eigenmodes purely structural. The effect of the inner fluid is still taken into account (corresponding to the acceleration term in equation (26)).

Under these assumptions the wave equation (26) reduces to:

$$
\frac{\partial^{2} \chi}{\partial t^{2}}-\frac{1}{\rho D} \frac{\partial^{2} \chi}{\partial x^{2}}+\frac{1}{\rho D} \frac{1}{K_{0}^{2}} \frac{\partial^{4} \chi}{\partial x^{4}}=0
$$


Using standard techniques, see Appendix B, it can be shown that the tube's natural modes of vibration are of two types:

$$
\begin{aligned}
& \tilde{\chi}_{n}^{(1)}(x)=\tanh \left(\frac{K_{n}^{(1)} L}{2}\right) \frac{\sin \left(k_{n}^{(1)} x\right)}{\cos \left(\frac{k_{n}^{(1)} L}{2}\right)}-\tan \left(\frac{k_{n}^{(1)} L}{2}\right) \frac{\sinh \left(K_{n}^{(1)} x\right)}{\cosh \left(\frac{K_{n}^{(1)} L}{2}\right)} \\
& \tilde{\chi}_{n}^{(2)}(x)=K_{n}^{(2)} \tanh \left(\frac{K_{n}^{(2)} L}{2}\right) \frac{\cos \left(k_{n}^{(2)} x\right)}{\cos \left(\frac{k_{n}^{(2)} L}{2}\right)}+k_{n}^{(2)} \tan \left(\frac{k_{n}^{(2)} L}{2}\right) \frac{\cosh \left(K_{n}^{(2)} x\right)}{\cosh \left(\frac{K_{n}^{(2)} L}{2}\right)}
\end{aligned}
$$

where $\left(k_{n}^{(1)}, K_{n}^{(1)}, \omega_{n}^{(1)}\right)$ and $\left(k_{n}^{(2)}, K_{n}^{(2)}, \omega_{n}^{(2)}\right)$ are the solutions of the following two sets of transcendental equations:

$$
\begin{aligned}
& \left\{\begin{array}{c}
\frac{k_{n}^{(1)} L}{2} \tanh \left(\frac{K_{n}^{(1)} L}{2}\right)=\frac{K_{n}^{(1)} L}{2} \tan \left(\frac{k_{n}^{(1)} L}{2}\right) \\
k_{n}^{(1)^{2}}=\frac{2 \pi}{D T_{S}}\left(\sqrt{1+\frac{T_{S} \rho D^{2} \omega_{n}^{(1)^{2}}}{\pi}}-1\right) \\
K_{n}^{(1)^{2}}=\frac{2 \pi}{D T_{S}}\left(\sqrt{1+\frac{T_{S} \rho D^{2} \omega_{n}^{(1)^{2}}}{\pi}}+1\right)
\end{array}\right. \\
& \left\{\begin{array}{c}
\frac{K_{n}^{(2)} L}{2} \tanh \left(\frac{K_{n}^{(2)} L}{2}\right)+\frac{k_{n}^{(2)} L}{2} \tan \left(\frac{k_{n}^{(2)} L}{2}\right)= \\
\frac{\omega_{n}^{(2)} \rho S_{S} L}{\left.-M \omega_{n}^{(2)}\right)^{2}+2 K_{a}}\left(\frac{K_{n}^{(2)}}{k_{n}^{(2)}}+\frac{k_{n}^{(2)}}{K_{n}^{(2)}}\right) \tanh \left(\frac{K_{n}^{(2)} L}{2}\right) \tan \left(\frac{k_{n}^{(2)} L}{2}\right) \\
k_{n}^{(2)}=\frac{2 \pi}{D T_{S}}\left(\sqrt{1+\frac{T_{S} \rho D^{2} \omega_{n}^{(2)}}{\pi}}-1\right) \\
K_{n}^{(2)^{2}}=\frac{2 \pi}{D T_{S}}\left(\sqrt{1+\frac{\left.T_{S} \rho D^{2} \omega_{n}^{(2)}\right)^{2}}{\pi}}+1\right)
\end{array}\right.
\end{aligned}
$$

The tube's modes of vibrations for the bulge motion can now be expressed 
using equation (25), which becomes:

$$
\begin{aligned}
& \tilde{S}_{n}^{(1)}(x)-S_{S}=-S_{S}\left(k_{n}^{(1)} \tanh \left(\frac{K_{n}^{(1)} L}{2}\right) \frac{\cos \left(k_{n}^{(1)} x\right)}{\cos \left(\frac{k_{n}^{(1)} L}{2}\right)}-K_{n}^{(1)} \tan \left(\frac{k_{n}^{(1)} L}{2}\right) \frac{\cosh \left(K_{n}^{(1)} x\right)}{\cosh \left(\frac{K_{n}^{(1)} L}{2}\right)}\right) \\
& \tilde{S}_{n}^{(2)}(x)-S_{S}=-S_{S} k_{n}^{(2)} K_{n}^{(2)}\left(-\tanh \left(\frac{K_{n}^{(2)} L}{2}\right) \frac{\sin \left(k_{n}^{(2)} x\right)}{\cos \left(\frac{k_{n}^{(2)} L}{2}\right)}+\tan \left(\frac{k_{n}^{(2)} L}{2}\right) \frac{\sinh \left(K_{n}^{(2)} x\right)}{\cosh \left(\frac{K_{n}^{(2)} L}{2}\right)}\right)
\end{aligned}
$$

Figure 3 shows the first six eigenmodes for the bulge motion, sorted by modal frequency, for the tube whose parameters are given in table (4). One can see that they behave like sine and cosine functions of spatial period $2 L / l$, where $l$ is an integer, except close to the tube ends where the boundary conditions impose zero amplitude for the bulge motion. The spatial period decreases with increasing modal frequency.

By comparing the tube's modes of vibrations to the general solution of [4] for the tube response in waves (equation (2.11) in [4]), one can see that there are two important differences. Firstly, modes of vibrations of the second type do not appear in the general solution of [4]. Indeed, they cannot exist if the tube is not allowed to move horizontally (which is the case in [4]). Secondly, the terms proportional to the hyperbolic functions in equation (29) do not appear in the solution of [4] because they don't take into account the longitudinal tension. In our study, taking into account the longitudinal tension is critical to achieve zero amplitude for the bulge motion at the tube ends (corresponding to the no-deformation condition, equation 23). 

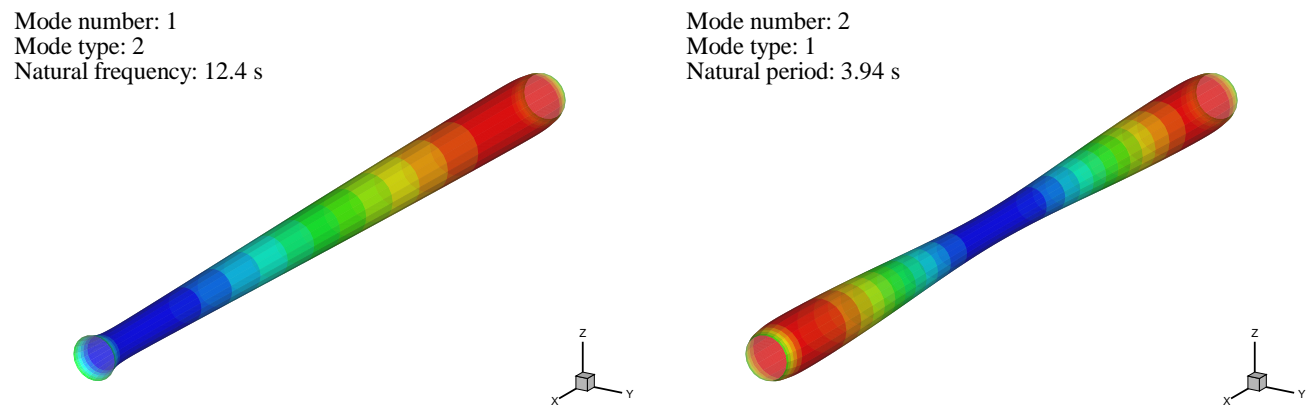

Mode number: 3
Mode type: 2

Natural period: $3.93 \mathrm{~s}$

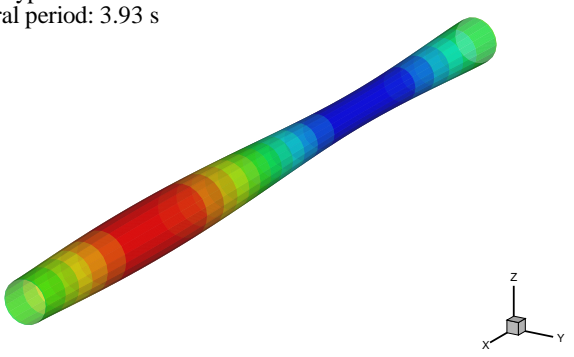

Mode number: 4

Mode type: 2

Natural frequency: $2.1 \mathrm{~s}$

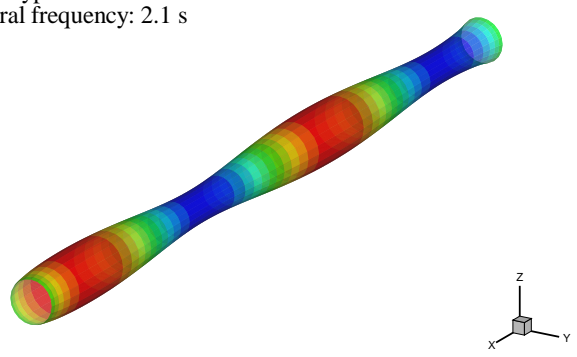

Mode number: 5

Mode type: 1

Natural period: $1.93 \mathrm{~s}$

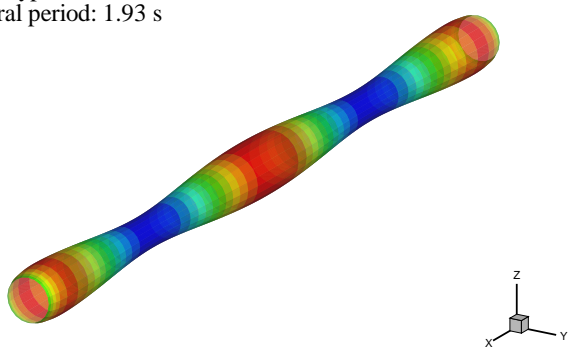

Mode number: 6

Mode type: 2

Natural period: $1.40 \mathrm{~s}$



Figure 3: Plot of the first six modes of deformation of the tube (ordered with respect to their natural frequencies) of the tube with parameters given in table 4 . It can be seen that the wavelength of the first mode is approximately equal to twice the tube length. It is approximately equal to the tube length for the second and third modes. It is half of the tube length for the fourth and fifth modes and one third of the tube length for the sixth mode. Note the rapid variation in tube deformation close to the tube ends, due to the zero deformation boundary conditions at the ends. 


\subsection{Tube response in waves}

Let us return to the full problem of the elastic tube response in waves $\left(p_{e x} \neq 0\right)$ with radiation effects $\left(p_{\text {rad }} \neq 0\right)$ and damping $\left(\eta \neq 0\right.$ and $\left.B_{R} \neq 0\right)$. For convenience of notation, let us define the assembled mode shape functions $\hat{\chi}_{m}$, which correspond to the set of modes of types (1) and (2), normalised and sorted by increasing natural frequencies.

Let us look for solutions that are time-dependent linear combinations of the mode shapes:

$$
\chi=\sum_{m=1}^{\infty} c_{m}(t) \hat{\chi_{m}}(x)
$$

Replacing $\chi$ in the wave equation (26) by this modal decomposition, multiplying by a mode $\hat{\chi}_{l}$ and integrating from $-\frac{L}{2}$ to $\frac{L}{2}$, one obtains:

$$
\sum_{m=1}^{\infty}\left(\begin{array}{c}
\ddot{c}_{m} \int_{-\frac{L}{2}}^{\frac{L}{2}} \hat{\chi}_{m} \hat{\chi}_{l} d x-\frac{1}{\rho D} c_{m} \int_{-\frac{L}{2}}^{\frac{L}{2}} \frac{\partial^{2} \hat{\chi}_{m}}{\partial x^{2}} \hat{\chi}_{l} d x \\
+\frac{1}{\rho D} \frac{1}{K_{0}^{2}} c_{m} \int_{-\frac{L}{2}}^{\frac{L}{2}} \frac{\partial^{4} \hat{\chi}_{m}}{\partial x^{4}} \hat{\chi}_{l} d x
\end{array}\right)=\left(\begin{array}{c}
-\frac{1}{\rho} \int_{-\frac{L}{2}}^{\frac{L}{2}} \frac{\partial \bar{p}_{e}}{\partial x} \hat{\chi}_{l} d x+ \\
\sum_{m=1}^{\infty} \dot{c}_{m} \int_{-\frac{L}{2}}^{\frac{L}{2}}\left(\eta \frac{\partial^{2} \hat{\chi}_{m}}{\partial x^{2}}-\frac{B_{R}}{S_{S}} \hat{\chi}_{m}\right) \hat{\chi}_{l} d x
\end{array}\right)
$$

Then, recalling the surge equation (18) and substituting the modal decomposition (30) and the wall equation in equation (20), one obtains:

$$
\begin{gathered}
M \ddot{X}+2 K_{a} X=-\Delta p_{e} S_{S}+\Delta p_{i} S_{S} \\
\left.M \sum_{m=1}^{\infty} \ddot{c}_{m} \hat{\chi}_{m}\right|_{\frac{L}{2}}+\left.2 K_{a} \sum_{m=1}^{\infty} c_{m} \hat{\chi}_{m}\right|_{\frac{L}{2}}-\frac{S_{S}}{D} \frac{1}{K_{0}^{2}} \sum_{m=1}^{\infty}\left(\left.\frac{\partial^{3} \tilde{\chi}_{m}}{\partial x^{3}}\right|_{\frac{L}{2}}-\left.\frac{\partial^{3} \tilde{\chi}_{m}}{\partial x^{3}}\right|_{-\frac{L}{2}}\right)=0
\end{gathered}
$$

Multiplying this last equation by $\left.\hat{\chi}_{l}\right|_{\frac{L}{2}}$, adding it to (31) and identifying the eigenmodes' orthogonality properties (B.23) and (B.26), one obtains a 
simple second order equation for the time evolution of the modal amplitudes:

$$
\begin{aligned}
& \ddot{c}_{m}+\omega_{m}^{2} c_{m}=-\frac{1}{\rho} \int_{-\frac{L}{2}}^{\frac{L}{2}} \frac{\partial \bar{p}_{e}}{\partial x} \hat{\chi}_{m} d x-\sum_{l=1}^{\infty}\left(\eta \epsilon_{l m}+\frac{B_{R}}{S_{S}} \varepsilon_{l m}\right) \dot{c}_{l} \\
& \ddot{c}_{m}+\omega_{m}^{2} c_{m}=\frac{1}{\rho} \int_{\frac{L}{2}}^{\frac{L}{2}} \bar{p}_{e} \frac{\partial \hat{\chi}_{m}}{\partial x} d x-\left.\Delta \bar{p}_{e} \hat{\chi}_{m}\right|_{\frac{L}{2}}-\sum_{l=1}^{\infty}\left(\eta \epsilon_{l m}+\frac{B_{R}}{S_{S}} \varepsilon_{l m}\right) \dot{c}_{l}(33)
\end{aligned}
$$

where

$$
\epsilon_{l m}=-\int_{-\frac{L}{2}}^{\frac{L}{2}} \frac{\partial^{2} \hat{\chi}_{m}}{\partial x^{2}} \hat{\chi}_{l} d x=\int_{-\frac{L}{2}}^{\frac{L}{2}} \frac{\partial \hat{\chi}_{m}}{\partial x} \frac{\partial \hat{\chi}_{l}}{\partial x} d x
$$

and

$$
\varepsilon_{l m}=\int_{-\frac{L}{2}}^{\frac{L}{2}} \hat{\chi}_{m} \hat{\chi}_{l} d x
$$

However, the outer pressure $\bar{p}_{e}$ remains to be described explicitly as a function of the incident wave parameters and the tube response. For each mode shape, a generalised radiation boundary value problem can be written:

$$
\left\{\begin{array}{cc}
\Delta \Phi_{m}=0 & \text { in } \Omega \\
\frac{\partial \Phi_{m}}{\partial n}=0 & \text { at } S_{b o t t o m} \\
\frac{\partial^{2} \Phi_{m}}{\partial t^{2}}+g \frac{\partial \Phi_{m}}{\partial z}=0 & \text { at } M \in S_{F S} \\
\frac{\partial \Phi_{m}}{\partial n}=-\frac{1}{2} r_{S} \frac{\partial \hat{\chi}_{m}}{\partial x} & \text { if at } \left.S_{B} \text { and if } x \in\right]-\frac{L}{2}, \frac{L}{2}[ \\
\frac{\partial \Phi_{m}}{\partial n}= \pm\left.\hat{\chi}_{m}\right|_{\frac{L}{2}} & \text { if at } S_{B} \text { and if } x= \pm \frac{L}{2}
\end{array}\right.
$$

Following [15], standard numerical codes based on Boundary Element Method (BEM) such as WAMIT [14] or NEMOH [2] can be tailored to solve this boundary value problem. Calculations are usually performed in the frequency domain. One output from these codes is the radiation pressure distribution $p_{m}(M, \omega)$ on the surface of the geometry, $\omega$ being the wave frequency. This is integrated over the contours of the tube $C(x)$ in order to provide standard added mass coefficients $A_{m}(x, \omega)=\Im\left(\frac{1}{\omega} \int_{C(x)} p_{m}(M) d x\right)$ and radiation 
damping coefficients $B_{m}(x, \omega)=-\Re\left(\int_{C(x)} p_{m}(M) d x\right)$. These coefficients allow the contour-averaged radiation pressure at coordinate $x$ along the tube to be related to the deformation velocity $\dot{c}_{m}$ and the acceleration $\ddot{c}_{m}$ :

$$
\bar{p}_{\text {rad }}(x, \omega)=\sum_{m=1}^{\infty}\left(-A_{m}(x, \omega) \ddot{c}_{m}-B_{m}(x, \omega) \dot{c}_{m}\right)
$$

BEM codes also allow calculation of the averaged excitation pressure complex coefficients $p_{e x}(x, \omega)$ resulting from the diffraction by the tube of an incident wave of unit amplitude and wave frequency $\omega$. Hence, the total hydrodynamic pressure reads, for an incident wave of unit amplitude:

$$
\bar{p}_{e}(x, t)=\bar{p}_{e x}(x, \omega)+\sum_{m=1}^{\infty}\left(-A_{m}(x, \omega) \ddot{c}_{m}-B_{m}(x, \omega) \dot{c}_{m}\right)
$$

Let us now define the mathematical operator ${ }^{\chi}$ (which is called $\chi$ transform in the following) as:

$$
\chi_{f}=\frac{1}{\rho} \int_{-\frac{L}{2}}^{\frac{L}{2}} f(x) \frac{\partial \hat{\chi}_{l}}{\partial x} d x-\left.\Delta f \hat{\chi}_{l}\right|_{\frac{L}{2}}
$$

where $f(x)$ is any arbitrary integrable function defined over $\left[-\frac{L}{2}, \frac{L}{2}\right]$ and $\Delta f=f\left(\frac{L}{2}\right)-f\left(-\frac{L}{2}\right)$.

Thus, the equation of motion (33) can be written in a simple matrix form:

$$
\left(\mathbf{I}+{ }^{\chi} \mathbf{A}\right) \ddot{\mathbf{C}}+\left({ }^{\chi} \mathbf{B}+\eta \boldsymbol{\epsilon}+\frac{B_{R}}{S_{S}} \boldsymbol{\varepsilon}\right) \dot{\mathbf{C}}+\omega^{2} \mathbf{C}={ }^{\chi} \mathbf{p}_{\text {ex }}
$$

where:

- $\mathbf{C}$ is the column vector of the $c_{l}$ coefficients.

- $\mathbf{I}$ is the matrix identity. 
- ${ }^{\chi} \mathbf{A}$ is the matrix of the ${ }^{\chi} A_{l m}$ frequency-dependent added mass coefficients.

- ${ }^{\chi} \mathbf{B}$ is the matrix of the ${ }^{\chi} B_{l m}$ frequency-dependent radiation damping functions.

- $\boldsymbol{\epsilon}$ is the matrix of the $\epsilon_{l m}$ wall damping coefficients.

- $\varepsilon$ is the matrix of the $\varepsilon_{l m}$ inner fluid damping coefficients.

- $\boldsymbol{\omega}$ is the diagonal matrix of the modal frequencies.

- ${ }^{\chi} \mathbf{p}_{\mathbf{e x}}$ is the column vector of the ${ }^{\chi} \bar{p}_{e x, l}$ excitation pressure coefficients.

It can be observed that radiation effects and damping create coupling between the modes (the off-diagonal terms of matrices ${ }^{\chi} \mathbf{A},{ }^{\chi} \mathbf{B}, \boldsymbol{\epsilon}$ and $\boldsymbol{\varepsilon}$ ) are non-zero).

Finally, by integrating over the tube surface the pressure difference across the tube wall times the radial deformation velocity and time-averaging, one can show that the mean power dissipated in the material is given by:

$$
P(\omega)=\frac{1}{2} \rho S_{S} \eta \sum_{m} \sum_{l} \Re\left(c_{m} c_{l}^{*}\right) \epsilon_{m l}
$$

\section{Experimental data}

Experiments on a model of this flexible electroactive wave energy converter were carried out in July 2011 in the large Hydrodynamics and Ocean Engineering Tank of Ecole Centrale de Nantes. The tank is 50 metres long, 30 metres wide and 5 metres deep. Waves are generated with 48 independently controlled flap wavemakers which allow generation of a large variety 


$\begin{array}{cccc}\text { Name } & \text { Symbol } & & \text { unit } \\ \text { Length } & L & 10 & \mathrm{~m} \\ \text { Radius } & r_{S} & 0.274 & \mathrm{~m} \\ \text { Thickness } & h_{S} & 0.01 & \mathrm{~m} \\ \text { Submergence } & z_{S} & -0.265 & \mathrm{~m} \\ \text { Tube mass } & M_{\text {tube }} & 91.7 & \mathrm{~kg} \\ \text { Towhead mass } & M_{\text {towhead }} & 110 . & \mathrm{kg} \\ \text { Mooring pretension } & F_{a S} & 443.4 & \mathrm{~N} \\ \text { Mooring stiffness } & K_{a} & 510 . & \mathrm{N} / \mathrm{m} \\ \text { Static pressure } & p_{S} & 77.7 & \mathrm{kPa} \\ \text { Yeoh model's parameter } & C_{10} & 134 & \mathrm{kPa} \\ \text { Yeoh model's parameter } & C_{20} & -22.2 & \mathrm{kPa} \\ \text { Yeoh model's parameter } & C_{20} & 7.30 & \mathrm{kPa} \\ \text { Distensibility } & D & 1.29 \times 10^{-4} & \mathrm{~Pa}-1 \\ \text { Material damping } & \eta & 0.323 & \mathrm{~m}^{2} . \mathrm{s}^{-1}\end{array}$

Table 1: Dimensions and parameters of the physical model

of regular, irregular and directional waves. Regular waves of heights up to 1 $\mathrm{m}$ can be generated in regular wave conditions.

The model is a 10-metre-long tube of diameter 0.42 metres at rest (not inflated), made of silicone and covered with Electroactive Polymer (EAP) sections. The other physical characteristics are listed in table 2 .

The tube is reinforced with Aramid fibres to avoid axial deformations. Twenty independent active sections were slipped onto the main tube (light stripes in figure 4) and kept in place due to the frictional effect once the 


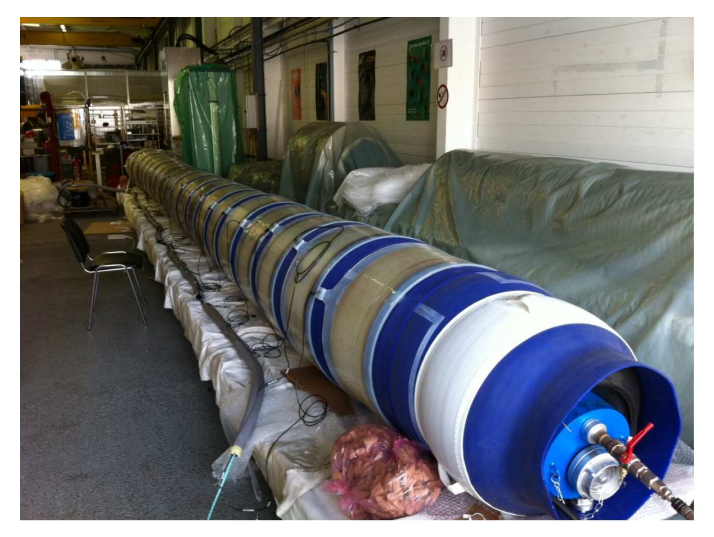

Figure 4: Experimental model during assembly



Figure 5: Underwater picture of the model during experiments. The model is below; the top image is a reflection in the free surface.

tube is inflated. They are made of multiple layers of electroactive polymer encapsulated in a silicone shell with waterproof electric connections to wire every section. Foam pads are inserted inside the tube to provide the necessary buoyancy to keep the system afloat.

The experimental model was moored at the centre of the basin by means of four anchor lines set nearly horizontal and attached to springs. The spring stiffness was chosen such that the natural period of the rigid body surge motion is much longer than that of tested waves (approximately 15 seconds).

Figure 5 shows an underwater picture of the model taken during the experiments. The bright rings are the active sections. The light points are LEDs that were fixed underneath the model using magnets, and were 
used as active markers to measure the $3 \mathrm{D}$ motion of the tube. Underwater cameras were placed on the bottom of the tank. Ad hoc software was used to calculate the motion of the marker from the images recorded by the cameras. Deformations of the tube can be seen on this snapshot: the ends are inflated whereas the middle is compressed.

In the laboratory tests of the Anaconda WEC described in [4], metal strain gauges were used. In our experiments, the electroactive sections were used. Indeed, every layers of the EAP has the property of changing its capacitance when deformed. The capacitance is proportional to a power of the deformation rate $\lambda=\frac{r}{r_{0}}$. During the tests, the capacitances were measured using a capacitance meter and their deformation rates were obtained from calibration tests carried out prior to tank testing.

Figure 6 shows measurements of the deformation rate $\lambda$ for section 5 ( $x=-2.75$ metres), 10 ( $x=-0.25$ metres $)$ and 20 ( $x=4.75$ metres $)$ for a regular wave of period 3.5 seconds and wave height approximately 0.2 metres. Transfer functions (also called Response Amplitude Operators, RAOs) for the variation of the radial deformation $\delta \lambda$ were defined as the variation of the tube radius around static equilibrium $\left(\delta \lambda=\frac{r}{r_{S}}-1\right)$. In order to remove any transient effect in their computation, the measurements were analyzed to identify a time window of five complete wave cycles over which the response appeared to be steady. For each section, the RAO (transfer function) was calculated as the amplitude of variation of the measurement (maximum minus minimum) divided by the measured amplitude of the incident wave.

For the purpose of validation, experimental measurements of deformations for regular waves of periods in the range 1 to 5 seconds and wave 

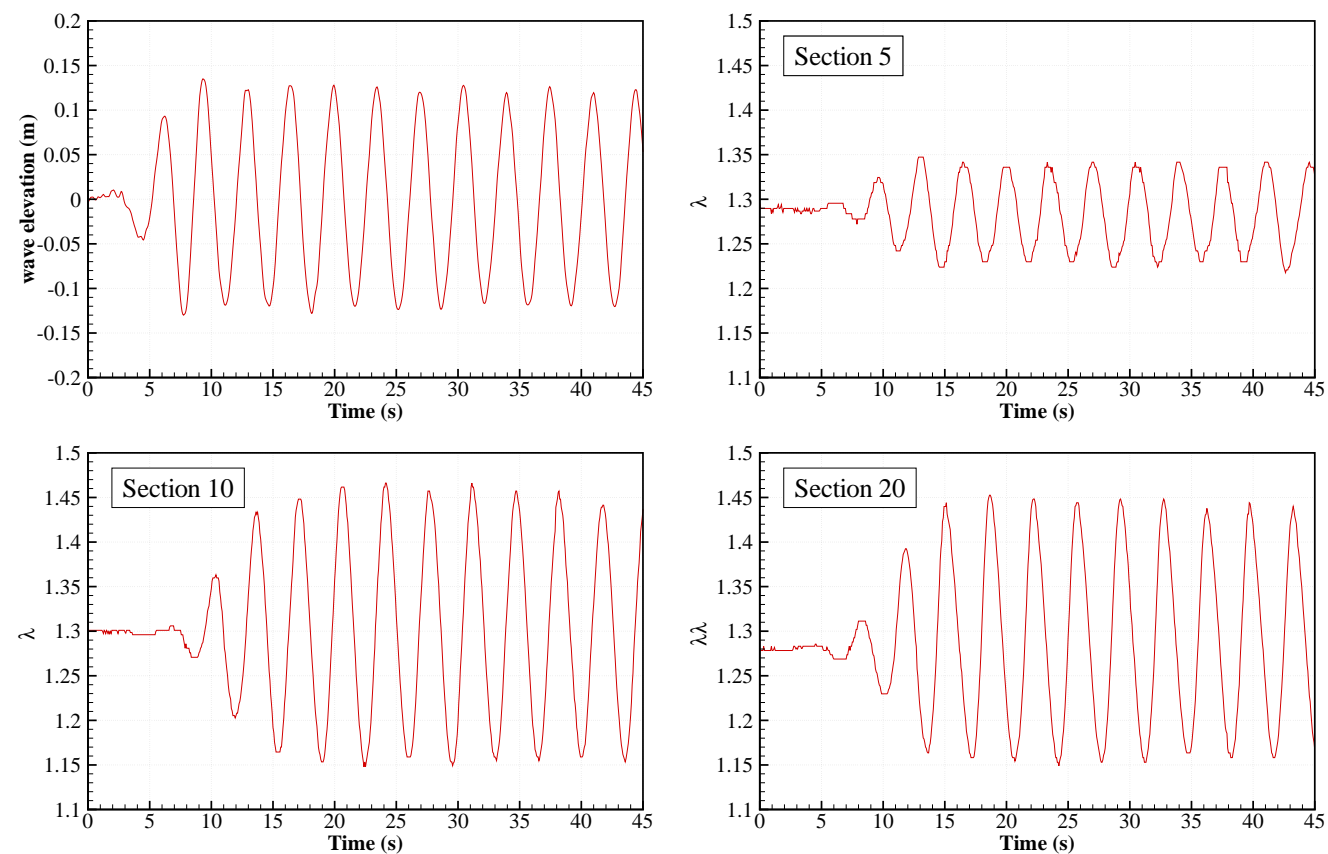

Figure 6: Measurements of deformation rate $\lambda=\frac{r}{r_{0}}$ for section 5 ( $x=-2.75$ metres), 10 ( $x=-0.25$ metres) and 20 ( $x=4.75$ metres $)$ for a regular wave of period 3.5 seconds and wave height approximately 0.2 metres. Top left figure is a measurement of the undisturbed incident wave elevation. 
height of 0.2 metres were used. Note that other conditions and measurements are available; experiments were also carried out for wave height of 0.4 metres, for irregular waves and for a shorter version of the tube with length of 7 metres. The inner and outer pressures were measured by twenty ceramic membrane pressure sensors distributed along the length of the tube, attached using magnets. Line tensions in the mooring lines and wave elevation at different locations in the tank were also measured using conventional equipment. Comparisons of numerical and experimental results for these other conditions is left for future work.

\section{Validation}

A suite of numerical tools was developed in Fortran to perform all the necessary computations needed to eventually solve the dynamic equation for the tube motion (40) including: a numerical tool for generating a mesh of the tube for the calculation of the hydrodynamic coefficients; a tool for calculating the coefficients in the equation of the mode shapes (29); and a tool for solving the equation of motion (40). For the calculation of the hydrodynamic coefficients ${ }^{\chi} \mathbf{A},{ }^{\chi} \mathbf{B}$ and ${ }^{\chi} \mathbf{p}_{\mathbf{e x}}$ in this last equation, the BEM code $\mathrm{NEMOH}[2]$ was used.

\subsection{Calculation of the hydrodynamic coefficients}

Figure 7 and figure 8 show respectively the diagonal coefficients of the added mass and radiation damping matrices ${ }^{\chi} \mathbf{A}$ and ${ }^{\chi} \mathbf{B}$; and the excitation pressure coefficients ${ }^{\chi} \mathbf{p}_{\text {ex }}$. In figure 7 , the coefficients are shown for two different discretizations of the tube geometry, from which it is clear that the 
calculations of the hydrodynamic coefficients converged. In the following, the discretization with 2548 panels was used. The mesh is shown in figure 9 .

It can be seen from figure 7 that the amplitude of the added mass coefficient ${ }^{\chi} \mathbf{A}$ is typically much smaller than 1 (remember that 1 are the inertia coefficients in the equation of motion 40). This is in agreement with [4] in which it was found that the surrounding water has a small effect for the case of a deeply submerged tube. It confirms the assumption from section 3.2 that radiation effects are small. This assumption was necessary to allow the analytical derivation of the mode shapes.

Note that a tube submergence of $z_{S}=1.25 r_{S}$ was used for the calculation of the hydrodynamic coefficients, whereas in the experiments described in section 4 it was floating almost underneath the free surface $\left(z_{S}=0.96 r_{S}\right.$ in the experiments). This is because mesh convergence could not be achieved for such small submergence - probably due to numerical issues for mesh panels that are almost horizontal and located close to the free surface. This issue was not further investigated because it is beyond the scope of this study. Instead, it was decided to use the smallest possible submergence for which it was possible to reach mesh convergence.

In order to assess the effect of the increased submergence on the hydrodynamic coefficients, we compared excitation pressure coefficients and FroudeKrylov pressure coefficients for different submergences. Froude-Krylov pressure coefficients are obtained by taking the ${ }^{\chi}$ transform of the pressure $p_{0}$ of the incident wave $\left(p_{0}(x, z)=i \rho g e^{k(z+i x)}\right)$ averaged on the contour $C(x)$. Figure 8 shows the excitation pressure coefficient for the first six modes for a submergence of $z_{S}=1.25 r_{S}$ and $z_{S}=1.5 r_{S}$ and the Froude-Krylov pres- 

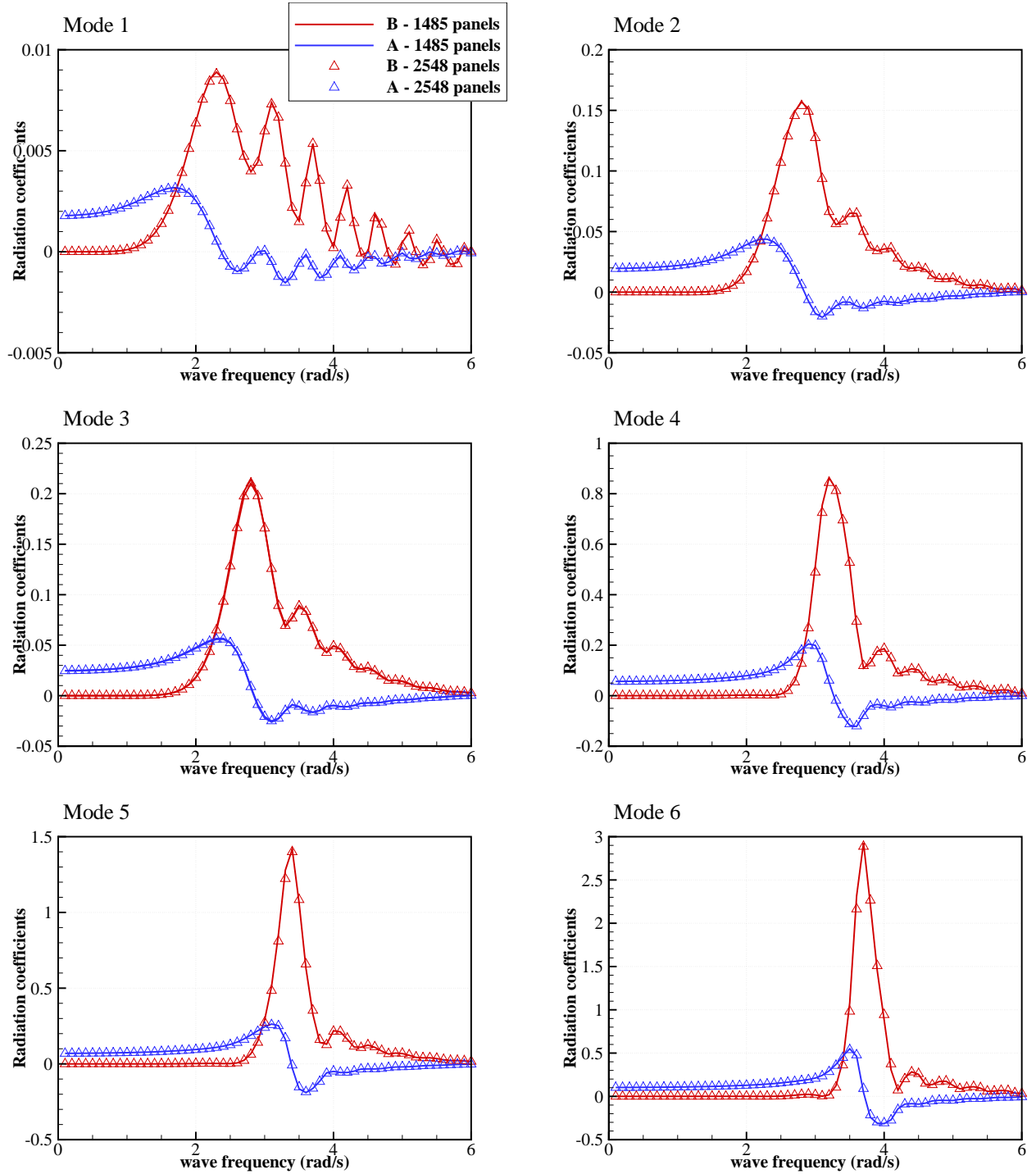

Figure 7: Six first diagonal coefficients of the added mass and radiation damping matrices

${ }^{\chi} \mathbf{A}$ and ${ }^{\chi} \mathbf{B}$ for two discretizations of the geometry. Note that the tube submergence is $z_{S}=1.25 r_{S}$ instead of $0.96 r_{S}$ in the experiments. 
sure coefficients ${ }^{\chi} \mathbf{p}_{\mathbf{0}}$ for the tube floating just underneath the free surface $\left(z_{S}=0.96 r_{S}\right)$ and for the submergence of $z_{S}=1.25 r_{S}$.

Comparing the Froude-Krylov pressure coefficients for the submergence used in the experiments $\left(z_{S}=0.96 r_{S}\right)$ with those for $z_{S}=1.25 r_{S}$, it can be seen that the difference is small: at most 10\% (see coefficients for modes 4 to 6). Comparing now the excitation pressure coefficients and the FroudeKrylov pressure coefficient for the submergence of $z_{S}=1.25 r_{S}$, it can be seen that the amplitude of the peak of the excitation pressure coefficient is approximately double that of the Froude-Krylov pressure coefficient, and the peak period is shifted towards lower frequencies. This implies that diffraction effects, which are taken into account in calculating the excitation pressure coefficient, are important. Note that significant diffraction effects for a horizontal tube were previously reported in [3].

In order to assess how the diffraction effects change with submergence, the excitation pressure coefficients for $z_{S}=1.5 r_{S}$ are also plotted in figure 8. By comparison with $z_{S}=1.25 r_{S}$, it can be seen that increasing the submergence has a significant effect on the amplitude of the coefficients: for modes 2 to 6 , the amplitude of the peak is reduced by 15 to $25 \%$. Extrapolating from this observation, the peak amplitude for the excitation pressure coefficient for the submergence used in the experiments $z_{S}=0.96 r_{S}$ may be 15 to $25 \%$ higher than for the submergence of $z_{S}=1.25 r_{S}$. In the following, which uses the results for $z_{S}=1.25 r_{S}$, an uncertainty of $20 \%$ should be retained for the hydrodynamic coefficients.

In figure 8 , for each mode the wave frequency whose wavelength is equal to the length of the mode is also indicated. Not surprisingly, it is close 

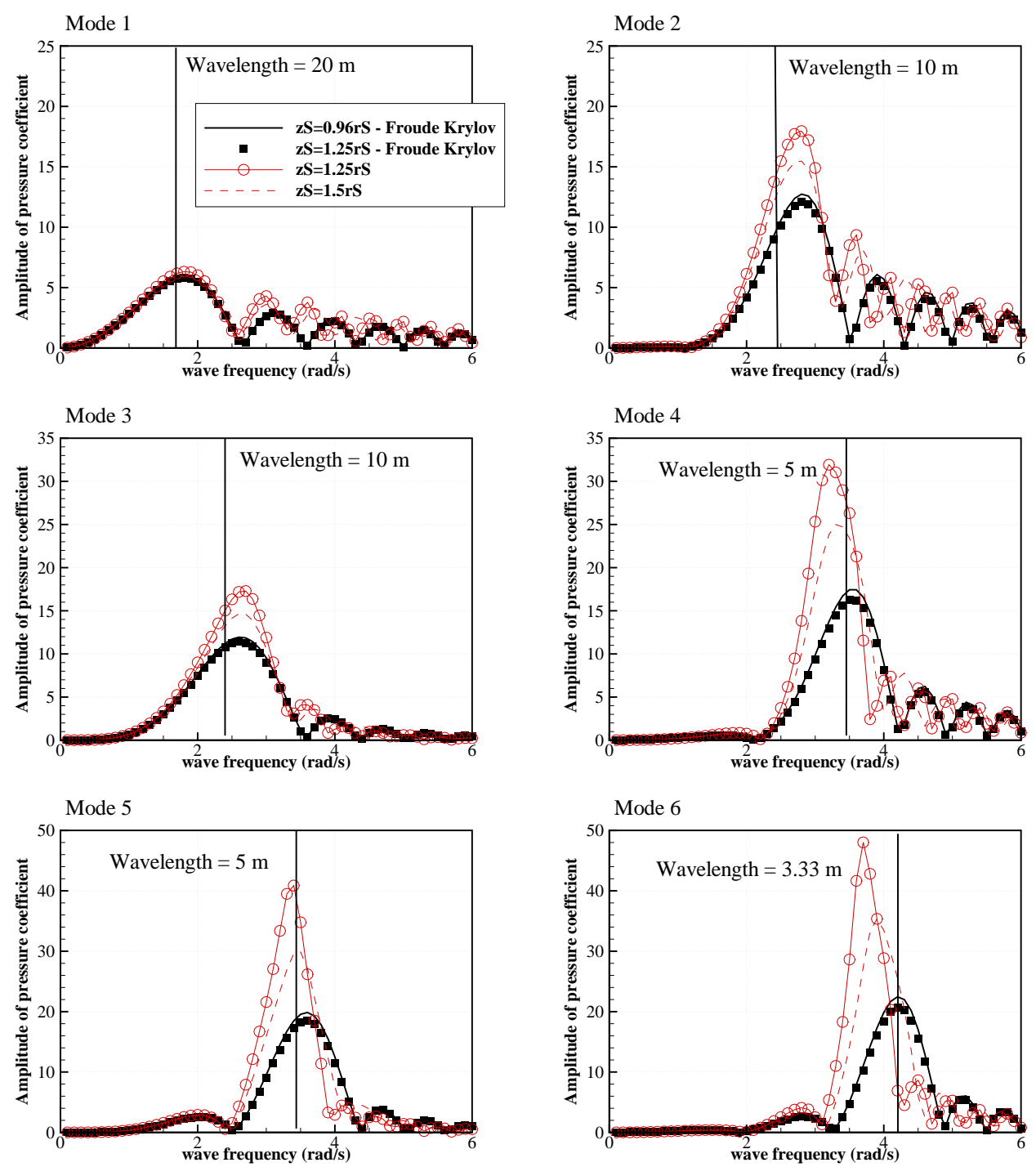

Figure 8: Excitation pressure coefficients ${ }^{\chi} p_{e x, i}$ for the first six modes of the tube for four different mesh discretizations. 


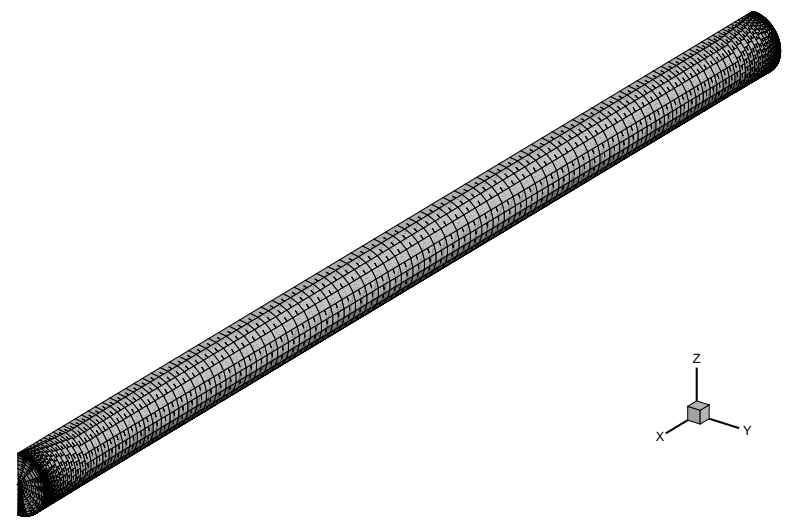

Figure 9: Mesh used for the calculation of the hydrodynamic coefficients $A_{m}(x, \omega)$, $B_{m}(x, \omega)$ and $p_{e x}(x, \omega)$. It has 2548 panels. Symmetry about the $(x O z)$ plane was used. The discretization is finer at the ends of the tube because of the rapid variation in the deformation at the ends (see figure 3 )

to the frequency of the maximum of the Froude-Krylov pressure coefficient. However, it can be seen that they don't match perfectly due to end effects (the tube deformation is 0 at both ends).

Figures 10 and 11 show snapshots of the free surface elevation for forced oscillations of the tube in modes 1 to 3 (figure 10) and 4 to 6 (figure 11). For each mode, the frequency was set equal to the modal frequency. One can see that the wave pattern becomes more complex as the mode number increases. Wave radiation also becomes increasingly directional and narrowcrested with increasing mode number. In particular, for modes 4 and 5 , it can be seen that the waves are essentially radiated in the $\mathrm{x}$-direction and that their width is of the order of the wavelength. For mode 6, four waves are radiated in a cross pattern. Again, the width of each wave appears to be of the order of the wavelength. From a wave energy conversion perspective, these 
multiple modes of radiation represent an interesting feature as they provide many different modes of interaction with the incident waves. However, it can be seen that overall the amplitude of the radiated wave is rather small. Large deformations will be needed to achieve a significant cancellation of the incident wave (and thus energy absorption [8]).

\subsection{Comparison of experimental and numerical results}

Figure 12 shows a comparison of experimental and numerical results for the tube's response (variation of the radial deformation $\delta \lambda$ per metre of wave amplitude) as a function of the wave period (vertical axis) and the tube section horizontal coordinate (horizontal axis). In the experiments, constant wave height of 0.2 metres was chosen for all wave periods. Thus, wave steepness ranged from $0.5 \%$ for the longest wave period up to $13 \%$ (almost the limit of wave breaking) for the shortest wave period. In the numerical simulations, the viscosity parameter $B_{R}$ was set to $18 \pi \nu$ corresponding to a one seventh power law for the flow velocity profile in the tube. The number of modes was truncated to 14, because it is sufficient to take into account those modes whose natural period is greater than 1 second.

In the experiments, resonance of the tube (large amplitude response) was observed in mode 2 for periods close to 3.5 seconds and in mode 4 for periods close to 2 seconds (see figure 3 for mode shapes). The periods of maximal response are in rough agreement with the modal periods provided in figure 3.

Overall, figure 12 shows there is reasonable agreement between the numerical and experimental results for deformation response. The numerical model is able to predict the tube response in mode 2 for periods between 3 

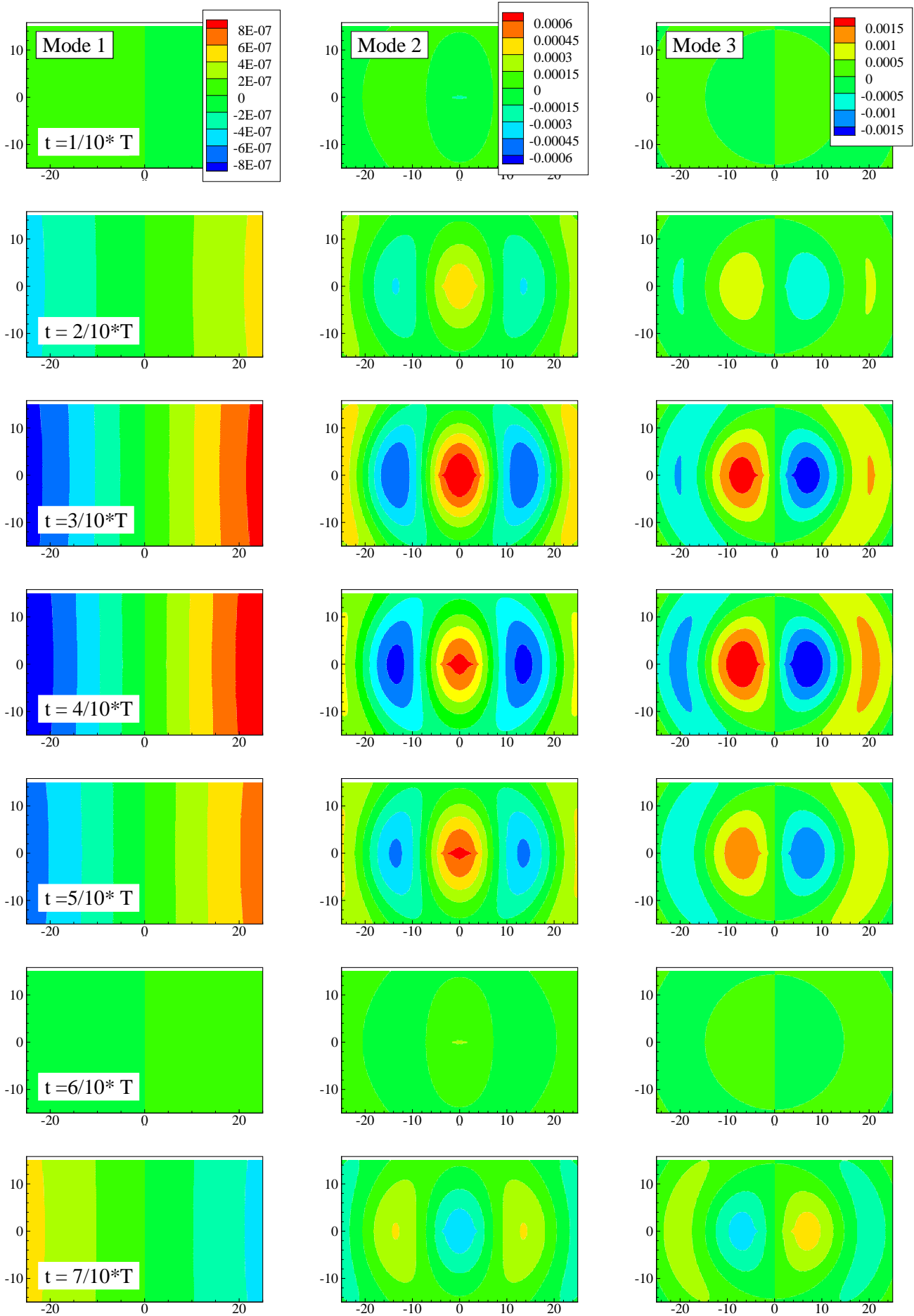

Figure 10: Snapshots of the wave elevations 5 seen from above for the radiated wave for modes 1 to 3 . For each mode, the period is equal to the modal period. 



Figure 11: Snapshots of the wave elevation segn from above for the radiated wave for 4 to 6 . For each mode, the period is equal to the modal period. 

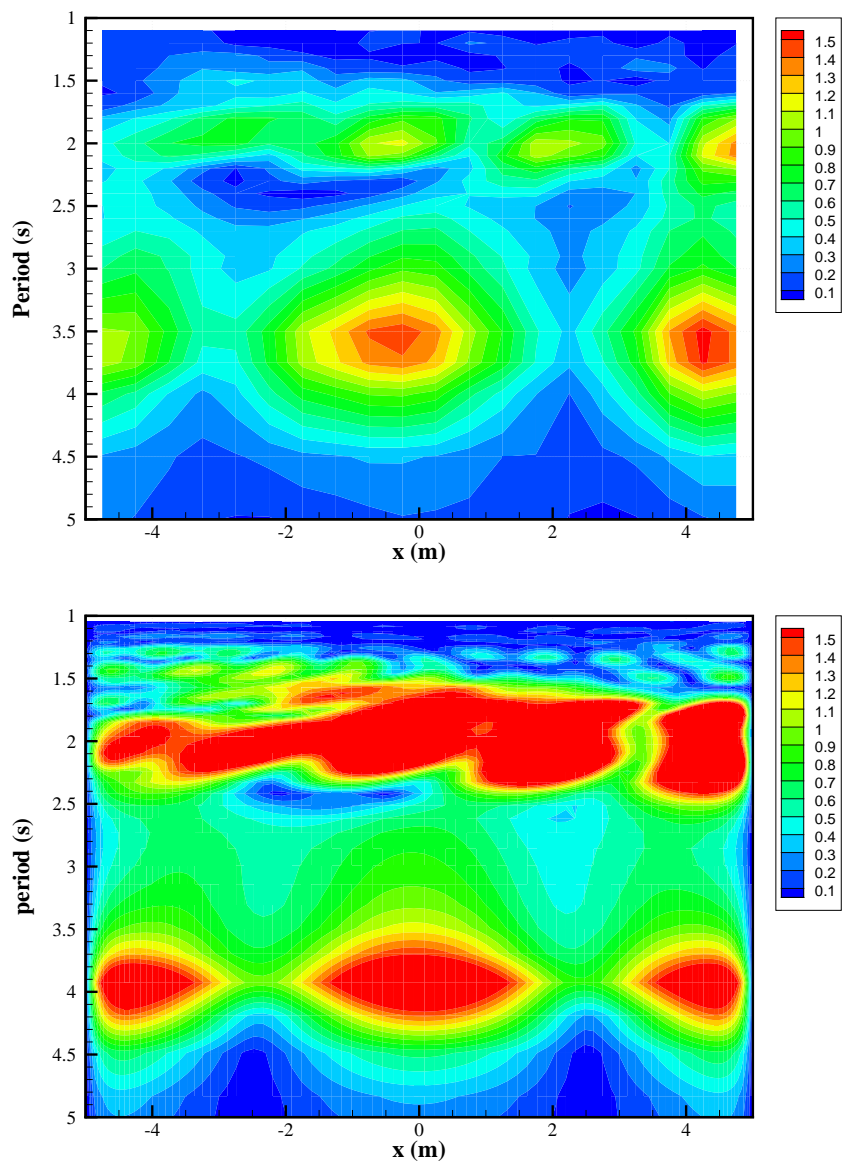

Figure 12: Comparison between experimental (top) and numerical (bottom) results for the variation of the radial deformation $\delta \lambda$ per metre of incident wave amplitude. Incident wave amplitude was 0.2 metres in the experiments. 
and 4.5 seconds and in mode 4 for periods close to 2 seconds. However, it is also clear that the numerical model considerably overestimates the response for periods close to resonances. Close inspection of the results also shows that the peak response in mode 3 has shifted from close to 3.5 seconds in the experiments to 4 seconds in the numerical results.

The theory of [4] predicts that, at resonance, the bulge wave in the tube can be written as the sum of a standing wave plus a progressive wave whose amplitude grows progressively as the wave travels along the tube. The experimental data in figure 12 agrees with this theory: the bulge amplitude is greater at the stern than at the bow of the tube and there are antinodes. Note that these effects are also present in the numerical data of figure 12 , altough the increase of the bulge amplitude along the tube cannot be seen because of the limited color scale.

In order to allow more quantitative comparisons between numerical and experimental results, figure 13 shows the deformation per metre of wave amplitude as a function of the wave period for the numerical model and the experiments at ten different sections along the tube. The experimental and numerical results show qualitative agreement. However, it can be seen that the resonance period predicted by the numerical model for mode 3 is approximately 0.5 seconds longer than that observed in the experiments.

A key parameter in determining the resonance period is the distensibility, $D$. Recall that there are significant uncertainties regarding this parameter: figure A.19 suggested that decreasing the material stiffness coefficient (which is inversely proportional to the distensibility) by up to a $20 \%$ may provide a better fit to the experimental data than using the value calculated from a 

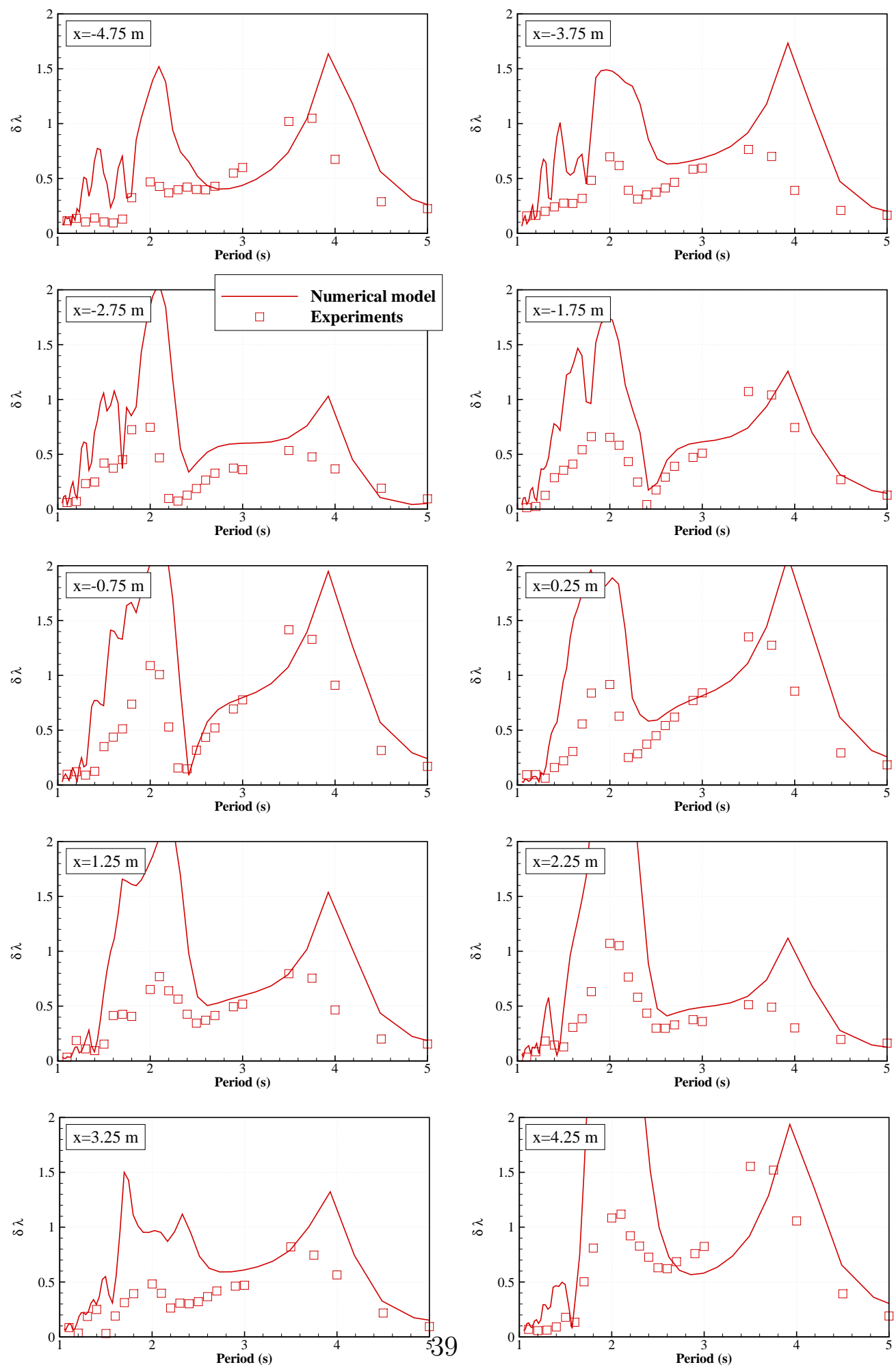

Figure 13: Comparison between experimental and numerical results for the variation of the deformation rate $\delta \lambda=\frac{r}{r_{S}}-1$ per metre of wave amplitude in regular waves at different sections along the tube. 
Taylor expansion of the Yeoh model (see Appendix A).

There is also substantial uncertainty in the wall damping coefficient $\eta$. Indeed, as explained in Appendix A, this damping coefficient was obtained by assuming the material damping process to be linear, and by matching the energy dissipated over a cycle to the linear model. Variation of the damping coefficient with amplitude and frequency of the cycle was not investigated in the experiments on the material. Thus, the wall damping may well be underestimated, and may vary with the frequency.

Consequently, the numerical model was re-run with the same parameters except for the distensibility and the wall damping coefficient. Five different values of distensibility were considered, ranging between $1.08 \times 10^{-7} \mathrm{~Pa}^{-1}$ and $1.29 \times 10^{-7} \mathrm{~Pa}^{-1}$ (thus in the range of uncertainty of the experimental data). The damping coefficient was varied between 1 and 3 times the experimental data. The overall agreement between the numerical results and the experiments was quantified by taking the average of the absolute error of the deformation over all sections and for periods between 3 and 5 seconds, where absolute error is defined to be the absolute value of the difference between the experimental and numerical results. Figure 14 shows the average error as a function of the distensibility and wall damping, from which it can be seen that the error is minimised for a distensibility of $1.12 \times 10^{-4} \mathrm{~Pa}^{-1}$ (a decrease of $15 \%$ ) and a wall damping coefficient of $0.581 \mathrm{~m}^{2} \cdot \mathrm{s}^{-1}$ (an increase of a factor almost 2).

The comparison between experimental and numerical results for the optimal material coefficients is shown in figure 15. Agreement between the models has clearly improved: it is now rather good for wave periods greater 


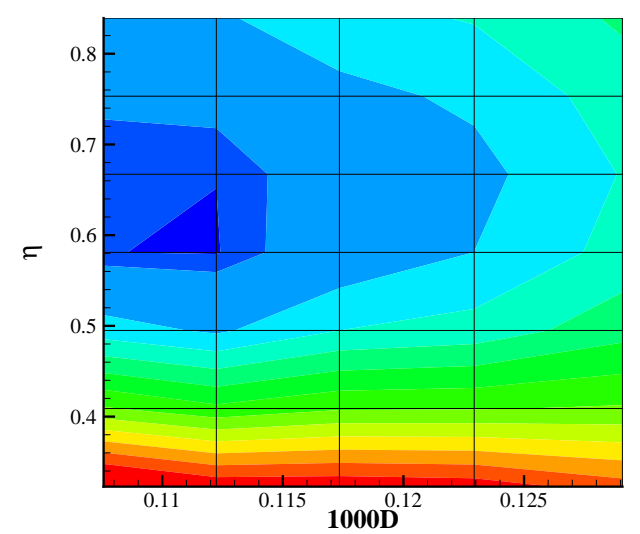

Figure 14: Average of the difference between the numerical and experimental results as a function of the distensibility $D$ and wall damping coefficient $\eta$.

than 2 seconds. However, for shorter periods, it appears that the numerical model still substantially overestimates the response. Recall that for periods in this range, the incident wave steepness in the experiments was in the range $3 \%$ to $13 \%$. Thus, these waves were non-linear, and since nonlinearities were not taken into account in the numerical model, this may explain the discrepancies observed. Dependence of the wall damping coefficient on wave period may also have a role.

Another possible source of underestimation of damping is the viscous damping coefficient $B_{R}$, which was estimated by assuming a one seventh power law for the velocity profile of the flow inside the tube. However, while this assumption may not be correct, it is nevertheless expected that the order of magnitude of the viscous damping coefficient $B_{R}$ is in the appropriate range. Numerical tests were performed with increasing $B_{R}$, from which it was found that it was necessary to increase $B_{R}$ by two order of magnitudes 



Figure 15: Comparison between experimental and numerical results for the vairation of the deformation $\delta \lambda$ in regular waves at different sections along the tube. The distensibility was taken to be $D=1.12 \times 10^{-4} \mathrm{~Pa}^{-1}$ and the wall damping to $\eta=0.581 \mathrm{~m}^{2} \cdot \mathrm{s}^{-1}$. One can see that the agreement between the numerical and experimental results is improved in comparison with figure 13. 
before there was any observable effect on the response of the tube. This shows that wall damping $\eta$, not viscous damping $B_{R}$, is the dominant source of damping in the system.

Although it was not quantified experimentally, it is believed that the metholodogy used largely underestimated the wall damping coefficient $\eta$. In future work, the ability of the numerical model to predict the tube response without tuning the material parameters may be improved by a more careful characterisation of this material parameter.

\section{Application to wave energy conversion}

In the experiments described in section 4 of this paper, rings of electroactive polymers were used as sensors for measuring the tube's deformation. Let us assume now that these rings are extracting energy (see [13] for more information on the electroactive material used and on how the rings are controlled to extract energy). It is assumed that the energy extraction effect on the tube dynamic response can be modelled as an additional wall stress term:

$$
\sigma_{P T O}=B_{P T O} \dot{S}
$$

$B_{P T O}$ is the so-called PTO (Power Take-Off) damping coefficient. PTO is the common term in the field of wave energy conversion for the system that converts the mechanical energy into electricity.

By incorporating the effect of the electroactive rings into equation (A.8), the model that has been presented in this paper can be used to study the energy performance of an energy-producing version of the S3 wave energy 
converter. The only modification required is to replace the coefficient $\eta$ in equation (40) by $\eta^{\prime}$ :

$$
\eta^{\prime}=\frac{h_{S} S_{S}\left(B_{m a t}+B_{P T O}\right)}{\rho r_{S}}
$$

The total power absorbed by the device (sum of power dissipated in the tube material plus sum of extracted power) is given by equation (41) by replacing $\eta$ by $\eta^{\prime}$. The net extracted power can be obtained by replacing $\eta$ by $\frac{h_{S} S_{S}}{\rho r_{S}} B_{P T O}$ :

$$
P(\omega)=\frac{1}{2} \frac{h_{S}}{r_{S}} S_{S}^{2} B_{P T O} \sum_{m} \sum_{l} \Re\left(c_{m} c_{l} *\right) \epsilon_{m l}
$$

Figure 16 shows results for the absorbed power of a wave energy converter whose dimensions and parameters are as described in section 4. Following the discussion in 5, the wall coefficients are set to $D=1.12 \times 10^{-4} \mathrm{~Pa}^{-1}$ and $\eta=0.581 \mathrm{~m}^{2} \cdot \mathrm{s}^{-1}$. The top figure shows only one graph for the absorbed power, because in this case the EAP rings are not active $\left(B_{P T O}\right.$ is set to 0$)$ - all the absorbed power is dissipated in the tube material. In the bottom figure, the EAP rings are producing electricity. It is assumed that their effect is such that $B_{P T O}=120 \mathrm{kPa} . \mathrm{s} / \mathrm{m}^{2}$. This value was optimized in order to achieve the highest peak in net extracted power in figure 16.

In this last figure, results are shown in non-dimensional form: the absorbed power is shown as the ratio of capture width $(\mathrm{CW})[7]$ to the tube length $L$. The capture width is defined as the ratio of absorbed power (in $\mathrm{kW})$ to the wave resource $J$ (in $\mathrm{kW} / \mathrm{m})$ :

$$
C W=\frac{P}{J}
$$





Figure 16: Non-dimensional absorbed power by the electroactive wave energy converter as a function of the non-dimensional wave period. In the top figure, the EAP rings are not active and the absorbed power is completely dissipated in the tube wall $\left(B_{P T O}=0\right)$. In the bottom figure, the EAP rings are active. It is assumed that their effect is such that $B_{P T O}=120 \mathrm{kPa} . \mathrm{s} / \mathrm{m}^{2}$. 
where the wave resource $J$ is given by $J=\frac{\rho g^{2} T}{8 \pi}$ for regular waves in deep water.

The unit of capture width is a length in metres. It may be interpreted as the width of wave crest that has been completely captured and absorbed by the WEC. A measure of the hydrodynamic efficiency of the system is further obtained by dividing the capture width by a characteristic dimension of the WEC. In this study, the tube length was used.

The top figure in figure 16 shows that, by interacting with the waves, the device can absorb and dissipate a width of wave crest in the range of 5 to 60 $\%$ of the tube length. It can also be seen that the device has a rather large bandwidth. However, it should be noted that the high peak power close to the non-dimensional period 2 is certainly overestimated. It corresponds to the peak response for wave periods close to 2 seconds in figure 15, for which the experimental response is roughly half of the numerical prediction of the numerical model. Therefore, for periods close 2 , it could be expected that the peak power might be four times smaller than shown in figure 16. Overall, it may be concluded that the non-active version of the device is able to dissipate a width of wave crest of 5 to $10 \%$ of its length for non-dimensional periods in the range 1.5 to 3.5 .

In the bottom figure in figure (16), the EAP rings are active and extracting energy. The bandwidth is similar to that of the non-active system. Again, the peak for non-dimensional periods close to 2 seconds is certainly overestimated, by a factor of around 4 . Nevertheless, it appears that in this case half of the absorbed power corresponds to wave power converted into electricity, the other half being dissipated in the material of the tube wall. The width 
of wave crest converted into electricity, one can see that it is approximately $5 \%$ of the tube length.

At sea, wave periods typicall range from a few seconds to a few tens of seconds, with 5-10 seconds being most frequently observed. Using Froude scaling, a scale of 10 would allow the tube response to match these most frequent wave conditions. This would yield a tube of length 100 metres, and the capture width could be expected to be close to 5 meters over a large range of wave periods. In deep water, mean annual wave resource is of the order of 20 to $40 \mathrm{~kW} / \mathrm{m}$ [12]. Thus, the annual mean power production of such a WEC may be of order 100 to $200 \mathrm{~kW}$.

However, note that theses figures should not be used to judge the potential performance of this wave energy converter concept. They are based on extrapolation from a particular design. Optimization of the dimensions and parameters may improve energy performance. Progress on the tube material may also reduce the amount of dissipated power, and thus enhance energy performance.

\section{Conclusion}

In this paper, a linear numerical model for analysing the dynamic response of a flexible Electroactive Wave Energy Converter is developed. It couples an inner flow problem with a structural problem and an outer flow problem. Under simplifying assumptions, the problem can be written in such a way that modal decomposition can be used. It allows the dynamic response of the tube to be described by a second order equation for the modal amplitudes that can easily be solved. 
The numerical model was implemented and numerical results are compared with experiments that were carried out on a 10-metre-long tube in a wave tank. Despite the simplifying assumptions, overall the model has a good ability to predict the WEC response to ocean waves, indicating that the assumptions were reasonable. Observations revealed that an accurate characterisation of the tube material stiffness and damping is critical for the accuracy of the model. Finally, Froude scaling was applied to extrapolate to the performance of a possible full-scale prototype suggesting that power absorption of the order of a few hundreds of kilowatts appears feasible for a 100-metre-long tube. Large energy losses are observed in the tube material. Reduction in these losses could significantly increase the net power production.

Though the numerical model shows a rather good ability to predict the WEC response to ocean waves, the model could be further improved by taking some of the non-linearities into account. Indeed, significant discrepancies were observed for short waves (for which wave steepness was large in the experiments). To improve accuracy, procedures for determining the material coefficients should be refined and it may be beneficial with respect to accuracy to take into account the non-linear stress-strain relation in the modelling of the tube wall response. The convection term in the momentum equation for the internal flow could also be taken into account, as could coupling with the vertical motions of the sections. The model could also be further validated against the other experimental results that are available.

Nevertheless, it is believed that the model proposed in this study is already useful for further investigation of the S3 WEC response to the waves, 

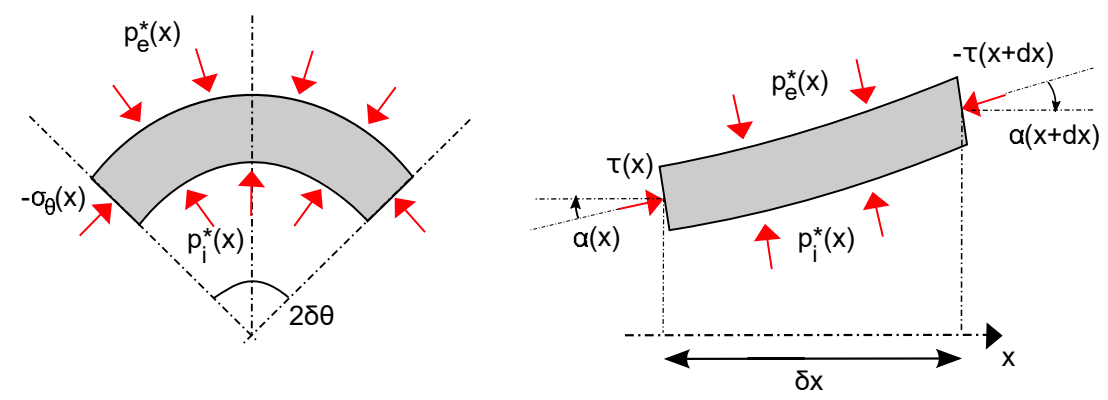

Figure A.17: Forces acting on a small piece of tube wall

or for optimization of its parameters in order to maximise power absorption while minimizing the costs. Hopefully, it will lead to a technical solution for wave energy conversion at a competitive cost of energy.

\section{Acknowledgements}

The financial support of the French Environment Agency ADEME through the funding scheme "Fond d'Investissement d'Avenir", project "S3", is gratefully acknowledged.

\section{Appendix A. Derivation of the linearised wall equation}

Let us consider a small piece of the tube wall of length $\delta x$ and width $r(x) \delta \theta$, as shown in figure A.17.

Let us assume that the tube mass is so small that inertia effects can be neglected. Then, at any time, the sum of all the forces acting on the small 
piece of tube shown in figure A.17 must be balanced:

$$
\begin{gathered}
-2 \sigma_{\theta} h \sin \left(\frac{\delta \theta}{2}\right) \delta x-p_{e}^{*}\left(r+\frac{h}{2}\right) \delta \theta \delta x+p_{i}^{*}\left(r-\frac{h}{2}\right) \delta \theta \delta x \\
-\tau(x+\delta x) h(x+\delta x) r(x+\delta x) \delta \theta \sin (\alpha(x+\delta x)) \\
+\tau(x) h(x) r(x) \delta \theta \sin (\alpha(x))=0
\end{gathered}
$$

In equation (A.1), $\sigma_{\theta}$ is the hoop stress in the wall, $\tau$ is the sum of the axial stress in the fibres reinforcing the tube and the stress in the wall in the axial direction, and $h$ is the thickness of the wall. Let us define the total longitudinal tensile force (wall tension) by $T(x)=2 \pi \tau(x) h(x) r(x)$. As a consequence of mass conservation and since the tube can not deform in the $x$ direction, one can show:

$$
h r=h_{S} r_{S}
$$

Let us consider only small deformations. Thus, $\sin \alpha=-\frac{\partial r}{\partial x}$ and $\cos \alpha=1$. Let us assume that $h$ is small with respect to $r$. Resolving forces vertically and retaining only the first order terms in equation (A.1), we obtain a relationship between the pressure difference across the tube wall, the hoop stress in the wall $\sigma_{\theta}$ and the total longitudinal pretension $T_{S}\left(=2 \pi r_{S} h_{S} \tau_{S}\right)$ in the reinforcing fibres.

$$
p_{e}^{*}-p_{i}^{*}=\frac{T_{S}}{2 \pi r_{S}} \frac{\partial^{2} r}{\partial x^{2}}-\frac{h}{r} \sigma_{\theta}
$$

By definition of $p_{i}$ and by using equation (A.2), it can be shown that:

$$
p_{i}=p_{e}-\frac{T_{S}}{2 \pi r_{S}} \frac{\partial^{2} r}{\partial x^{2}}+\frac{h_{S}}{r_{S}} \frac{S_{S}}{S} \sigma_{\theta}-p_{i S}
$$

At rest, it is assumed that the tube is not deformed, i.e $\frac{\partial^{2} r}{\partial x^{2}}(x)=0$. Thus, the static hoop stress in the wall and the static pressure must be balanced: 
$\frac{h_{S}}{r_{S}} \sigma_{\theta S}=p_{i S}$, where $\sigma_{\theta S}$ is the hoop stress at equilibrium. Hence, equation (A.4) can be rewritten as:

$$
p_{i}=p_{e}-\frac{T_{S}}{2 \pi r_{S}} \frac{\partial^{2} r}{\partial x^{2}}+\frac{h_{S}}{r_{S}}\left(\frac{S_{S}}{S} \sigma_{\theta}-\sigma_{\theta S}\right)
$$

By averaging this last equation over the contour $C(x)$ of the section $S(x)$ (i.e. the circle $r=r(x))$ and by using the fact that to first order $\frac{1}{r_{S}} \frac{\partial^{2} r}{\partial x^{2}}=\frac{1}{2 S_{S}} \frac{\partial^{2} S}{\partial x^{2}}$ :

$$
p_{i}=\bar{p}_{e}-\frac{T_{S}}{4 \pi S_{S}} \frac{\partial^{2} S}{\partial x^{2}}+\frac{h_{S}}{r_{S}}\left(\frac{S_{S}}{S} \sigma_{\theta}-\sigma_{\theta S}\right)
$$

where $\bar{p}_{e}(x)=\int_{C(x)} p_{e}(M) d C$ is the contour-averaged outer pressure.

\section{Material behaviour}

In the experiments described in section 4 , the tube was made of elastic material. The behaviour of this material is assumed to be visco-hyperelastic.

The hyperelastic behaviour was investigated through dedicated experiments. Samples of the tube material were tested in order to measure the force as a function of the deformation in quasi-static conditions. It was further assumed that the material behaviour follows the Yeoh model [18] so that the stress for planar extension $\sigma_{h e l}$ can be written:

$$
\sigma_{\text {hel }}=2\left(\lambda^{2}-\frac{1}{\lambda^{2}}\right)\left(C_{10}+2 C_{20}\left(I_{1}-3\right)+3 C_{30}\left(I_{1}-3\right)^{2}\right)
$$

where $I_{1}=\left(\lambda^{2}+\frac{1}{\lambda^{2}}+1\right) . \lambda=\frac{r}{r_{0}}=\frac{h_{0}}{h}$ is the deformation rate where $r_{0}$ and $h_{0}$ are the radius and thickness at rest (tube not inflated).

The material parameters $C_{10}, C_{20}, C_{30}$ were obtained by least square fitting of the Yeoh model to the experimental results. It was found $C_{10}=134$ $\mathrm{kPa}, C_{20}=-22.2 \mathrm{kPa}$ and $C_{30}=7.30 \mathrm{kPa}$. 




Figure A.18: Measurement of stress in a sample of the tube material when cyclic deformations are applied. Hysteresis is observed. The area of the hysteresis loop corresponds to energy that is dissipated due to internal friction in the material.

The effect of material viscosity was also investigated experimentally. Cyclic deformations were applied to a sample of the material. The stress was recorded as a function of the strain. The results exhibited hysteresis (see figure A.18). The area of the hysteresis loop corresponds to energy that was dissipated due to internal friction.

In a first approach, energy dissipation is modelled by an additional linear damping term $\sigma_{v i s}=B_{v i s} \dot{\lambda}$ in the wall stress $\sigma_{\theta}$. The material damping coefficient $B_{v i s}$ was obtained by matching the energy dissipated over a cycle with the experiments. It was found that $B_{v i s}=17.8 \mathrm{kPa} . \mathrm{s}$.

Finally, the total stress $\sigma_{\theta}$ is assumed to be the sum of the hyperelastic stress $\sigma_{h e l}$ and the viscous stress $\sigma_{v i s}$ : 


$$
\sigma_{\theta}=\sigma_{h e l}+\sigma_{v i s}
$$

\section{Linearised wall equation}

In the experiments described in section 4 , the static deformation at equilibrium $\lambda_{S}$ was 1.309. Let us define the term $\frac{S_{S}}{S} \sigma_{\theta}-\sigma_{\theta S}$ in equation (A.6) to be:

$$
\frac{S_{S}}{S} \sigma_{\theta}-\sigma_{\theta S}=K_{m a t}\left(S-S_{S}\right)+B_{m a t} \dot{S}+\delta_{\sigma}(S)
$$

where $\delta_{\sigma}(S)$ is a correction function, $K_{m a t}$ is an ad-hoc coefficient representing wall stiffness, and $B_{m a t}=\frac{1}{2 S_{S}} B_{v i s}$ is the wall damping. According to Taylor expansion, the correction function is of order $O\left(S-S_{S}\right)^{2}$ in the vicinity of $S=S_{S}$ by choosing $K_{m a t}$ such as:

$$
\begin{aligned}
K_{\text {mat }}=\frac{4}{S_{S}}\left\{\frac{1}{\lambda_{S}^{2}}\left[C_{10}+2 C_{20}\left(I_{1 S}-3\right)+3 C_{30}\left(I_{1 S}-3\right)^{2}\right]\right. \\
\left.+\left(\lambda_{S}^{2}-\frac{1}{\lambda_{S}^{2}}\right)\left[C_{20}+3 C_{30}\left(I_{1 S}-3\right)\right]\right\}
\end{aligned}
$$

with $I_{1 S}=\left(\lambda_{S}^{2}+\frac{1}{\lambda_{S}^{2}}+1\right)$.

Figure A.19 shows a comparison of the internal pressure as a function of the cross-sectional area measured during the inflation of the tube compared to the pressure predicted by the different models. It can be seen that the pressure predicted by the Yeoh model has rather good agreement with measurement, particularly taking into account that the parameters of the Yeoh model were derived from experiments on a small sample of the tube material and not the complete tube (as explained in the previous section). 
However, for cross-sectional area close to static equilibrium $\left(S_{S} \approx 0.235\right.$ $\mathrm{m}^{2}$ ), the uncertainty in the pressure measurement appears to be significant. In figure A.19, internal pressure as function of the section has been plotted for the linear model with coefficient $K_{\text {mat }}$ calculated according to equation (A.10) (dotted line) and with a 10\% larger coefficient (dashed-dotted line) and $20 \%$ larger coefficient. Visually, it is not obvious which of the three models provides the best match with the pressure measurements. It shows that there are significant uncertainties in the spatial derivative of the stress as function of the deformation close to static equilibrium. It is noteworthy, because it is believed to be one major explanation for discrepancies between numerical and experimental results, as it is discussed in section 5 .

Combining equations (A.6) and (A.9), the following wall equation is obtained:

$$
p_{i}=\bar{p}_{e}-\frac{1}{D S_{S}} \frac{1}{K_{0}^{2}} \frac{\partial^{2} S}{\partial x^{2}}+\frac{1}{D S_{S}}\left(S-S_{S}\right)+\frac{1}{D S_{S} K_{m a t}} \delta_{\sigma}+\frac{\rho}{S_{S}} \eta \dot{S}
$$

where $D=\frac{r_{S}}{h_{S} S_{S} K_{m a t}}, \frac{1}{K_{0}^{2}}=\frac{T_{S} D}{4 \pi}$ and $\eta=\frac{h_{S} S_{S} B_{m a t}}{\rho r_{S}} . D$ is usually referred to as the distensibility [9].

For small deformations around the static equilibrium, the correction $\delta_{\sigma}$ is negligible, leading to the following linearised wall equation:

$$
p_{i}=\bar{p}_{e}-\frac{1}{D S_{S}} \frac{1}{K_{0}^{2}} \frac{\partial^{2} S}{\partial x^{2}}+\frac{1}{D S_{S}}\left(S-S_{S}\right)+\frac{\rho}{S_{S}} \eta \dot{S}
$$




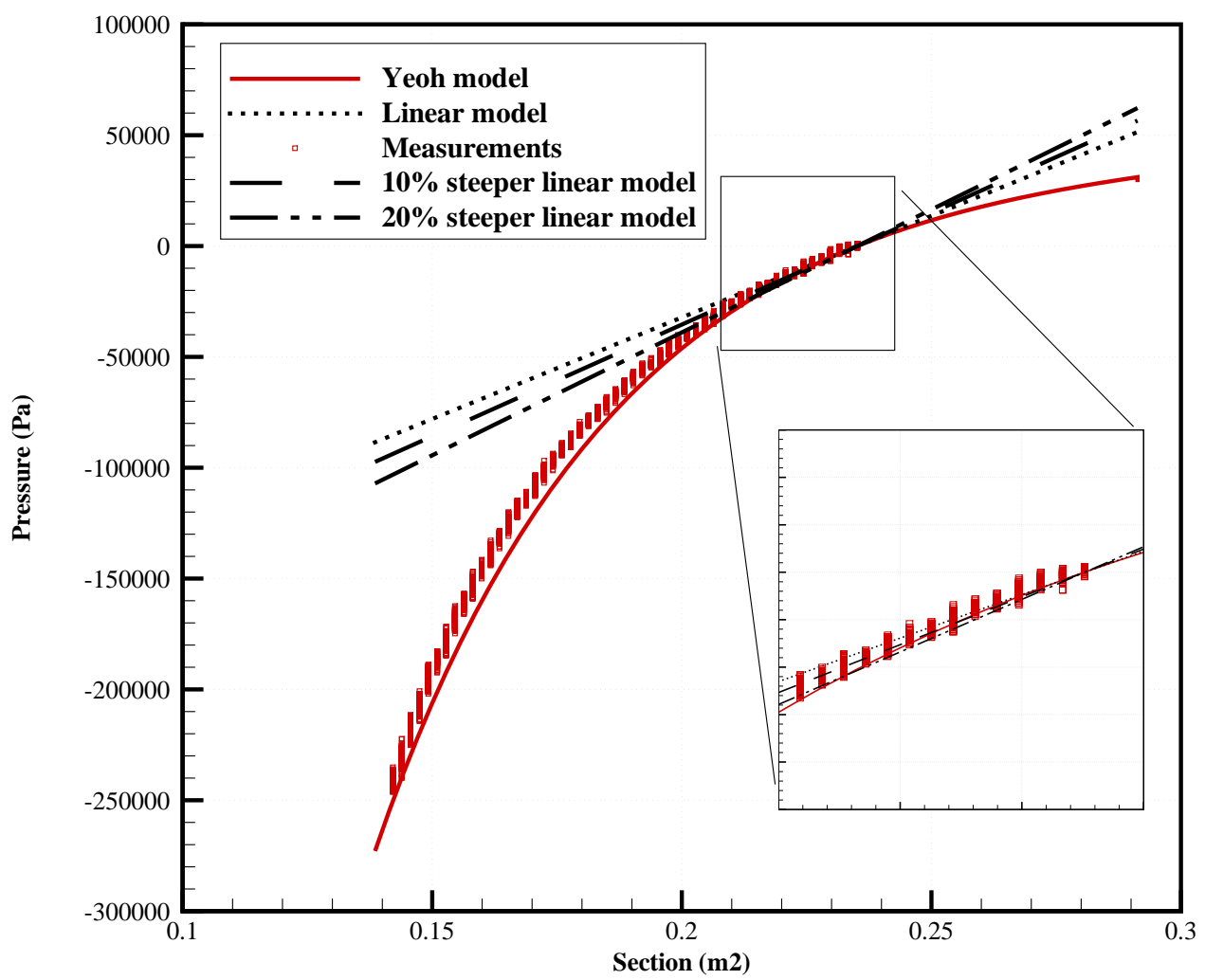

Figure A.19: Comparison of internal pressure in the tube as a function of its cross-sectional area measured during inflation compared to the pressure predicted by the different models. Solid line is for the Yeoh model (equation (A.7)), dotted line is the linear model with stiffness coefficient $K_{\text {mat }}$ calculated according to equation (A.10), dashed-dotted line is the linear model with a $10 \%$ larger stiffness coefficient and dashed dotted line is for the linear model with a $20 \%$ larger coefficient. For cross-sectional area close to static equilibrium, one can see that it is not obvious which of the three linear models provides the best match with measurements. This shows there is uncertainty in the spatial derivative of the stress as function of the deformation. 


\section{Appendix B. Eigenmodes of wave equation (27)}

Derivation of the eigenmodes

Let us look for the eigen solutions of the wave equation:

$$
\frac{\partial^{2} \chi}{\partial t^{2}}-\frac{1}{\rho D} \frac{\partial^{2} \chi}{\partial x^{2}}+\frac{1}{\rho D} \frac{1}{K_{0}^{2}} \frac{\partial^{4} \chi}{\partial x^{4}}=0
$$

Let us look for solutions of the form:

$$
\chi(x, t)=\Re\left(\tilde{\chi} e^{i \gamma x} e^{-i \omega t}\right)
$$

in which $\gamma$ and $\omega$ are the wave number and the wave frequency, respectively. Substituting equation (B.2) into the wave equation, it follows that $\gamma$ and $\omega$ must be related according to:

$$
-\omega^{2}+\frac{1}{\rho D} \gamma^{2}+\frac{1}{\rho D} \frac{1}{K_{0}^{2}} \gamma^{4}=0
$$

The solutions of this last equation are:

$$
\begin{aligned}
& \gamma_{1}^{2}=\frac{2 \pi}{D T_{S}}\left(\sqrt{1+\frac{T_{S} \rho D^{2} \omega^{2}}{\pi}}-1\right) \\
& \gamma_{2}^{2}=-\frac{2 \pi}{D T_{S}}\left(\sqrt{1+\frac{T_{S} \rho D^{2} \omega^{2}}{\pi}}+1\right)
\end{aligned}
$$

from which:

$$
\gamma_{1}= \pm k, \quad \gamma_{2}= \pm i K
$$

with $k$ and $K$ positive real numbers. Thus, general solutions of the wave equation (27) are of the form:

$$
\tilde{\chi}(x)=A^{+} e^{i k x}+A^{-} e^{-i k x}+B^{+} e^{K x}+B^{-} e^{-K x}
$$


Now let us consider the boundary conditions at the ends of the tube. The inner fluid velocity must be equal to the surge velocity of the tube for $x= \pm \frac{L}{2}$, equation 22 , leading to:

$$
\begin{aligned}
& A^{+} e^{-i k \frac{L}{2}}+A^{-} e^{i k \frac{L}{2}}+B^{+} e^{-K \frac{L}{2}}+B^{-} e^{K \frac{L}{2}}=\tilde{X} \\
& A^{+} e^{i k \frac{L}{2}}+A^{-} e^{-i k \frac{L}{2}}+B^{+} e^{K \frac{L}{2}}+B^{-} e^{-K \frac{L}{2}}=\tilde{X}
\end{aligned}
$$

where $\tilde{X}$ such that $X(t)=\Re\left(\tilde{X} e^{-i \omega t}\right)$.

The two ends of the tube can not deform (23). Thus:

$$
\begin{aligned}
& i k A^{+} e^{-i k \frac{L}{2}}-i k A^{-} e^{i k \frac{L}{2}}+K B^{+} e^{-K \frac{L}{2}}-K B^{-} e^{K \frac{L}{2}}=0 \\
& i k A^{+} e^{i k \frac{L}{2}}-i k A^{-} e^{-i k \frac{L}{2}}+K B^{+} e^{K \frac{L}{2}}-K B^{-} e^{-K \frac{L}{2}}=0
\end{aligned}
$$

By manipulating these last four equations, one can show:

$$
\begin{aligned}
\left(A^{+}+A^{-}\right) \cos \left(\frac{k L}{2}\right)+\left(B^{+}+B^{-}\right) \cosh \left(\frac{K L}{2}\right) & =-\tilde{X} \\
i\left(A^{+}-A^{-}\right) \sin \left(\frac{k L}{2}\right)+\left(B^{+}-B^{-}\right) \sinh \left(\frac{K L}{2}\right) & =0 \\
i k\left(A^{+}-A^{-}\right) \cos \left(\frac{k L}{2}\right)+K\left(B^{+}-B^{-}\right) \cosh \left(\frac{K L}{2}\right) & =0 \\
-k\left(A^{+}+A^{-}\right) \sin \left(\frac{k L}{2}\right)+K\left(B^{+}+B^{-}\right) \sinh \left(\frac{K L}{2}\right) & =0
\end{aligned}
$$

Using equation (19), $\tilde{X}$ can be related to the coefficients $A^{ \pm}$and $B^{ \pm}$. Since it has been assumed that the dynamic pressure in the outer fluid is equal to $0, \bar{p}_{e}=0$, it follows that:

$$
\begin{aligned}
0 & =M \ddot{X}+2 K_{a} X+\rho S_{S} \int_{-\frac{L}{2}}^{\frac{L}{2}} \frac{\partial U}{\partial t} d x \\
\tilde{X} & =\frac{2 \omega^{2} \rho S_{S}}{-M \omega^{2}+2 K_{a}}\left(\frac{1}{k}\left(A^{+}+A^{-}\right) \sin \left(\frac{k L}{2}\right)+\frac{1}{K}\left(B^{+}+B^{-}\right) \sinh \left(\frac{K L}{2}\right)\right)
\end{aligned}
$$


Finally, equations (B.11) and (B.12) can be assembled to form a set of two linear systems:

$$
\begin{array}{r}
{\left[\begin{array}{cc}
\cos \left(\frac{k L}{2}\right)-\kappa \frac{2}{k L} \sin \left(\frac{k L}{2}\right) & \cosh \left(\frac{K L}{2}\right)-\kappa \frac{2}{K L} \sinh \left(\frac{K L}{2}\right) \\
-k \sin \left(\frac{k L}{2}\right) & K \sinh \left(\frac{K L}{2}\right)
\end{array}\right]\left[\begin{array}{l}
A^{+}+A^{-} \\
B^{+}+B^{-}
\end{array}\right]=\left[\begin{array}{l}
0 \\
0
\end{array}\right]} \\
{\left[\begin{array}{cc}
i k \cos \left(\frac{k L}{2}\right) & K \cosh \left(\frac{K L}{2}\right) \\
i \sin \left(\frac{k L}{2}\right) & K \sinh \left(\frac{K L}{2}\right)
\end{array}\right]\left[\begin{array}{l}
A^{+}-A^{-} \\
B^{+}-B^{-}
\end{array}\right]=\left[\begin{array}{l}
0 \\
0
\end{array}\right]}
\end{array}
$$

with $\kappa=\frac{\omega^{2} \rho S_{S} L}{-M \omega^{2}+2 K_{a}}$. There are non-trivial solutions only if one of the following sets of conditions is fulfilled:

$$
(S 1)\left\{\begin{array}{c}
\frac{k L}{2} \tanh \left(\frac{K L}{2}\right)=\frac{K L}{2} \tan \left(\frac{k L}{2}\right) \\
\left(A^{+}-A^{-}\right) i \sin \left(\frac{k L}{2}\right)+\left(B^{+}-B^{-}\right) \sinh \left(\frac{K L}{2}\right)=0 \\
A^{+}=-A^{-} \\
B^{+}=-B^{-}
\end{array}\right.
$$

or

$$
S 2)\left\{\begin{array}{c}
\frac{K L}{2} \tanh \left(\frac{K L}{2}\right)+\frac{k L}{2} \tan \left(\frac{k L}{2}\right)-\kappa\left(\frac{K}{k}+\frac{k}{K}\right) \tanh \left(\frac{K L}{2}\right) \tan \left(\frac{k L}{2}\right)=0 \\
-k\left(A^{+}-A^{-}\right) \sin \left(\frac{k L}{2}\right)+ \\
\left.A^{+}=B^{-}-B^{-}\right) \sinh \left(\frac{K L}{2}\right)=0 \\
B^{+}=B^{-}
\end{array}\right.
$$

There are many combinations of $k, K$ and $\omega$ which are solutions of the first equation in either $(S 1)$ or $(S 2)$. Let $k_{n}^{(1)}, K_{n}^{(1)}$ and $\omega_{n}^{(1)}$ denote the $n^{\text {th }}$ solution of $(S 1)$ and $k_{n}^{(2)}, K_{n}^{(2)}$ and $\omega_{n}^{(2)}$ the $n^{\text {th }}$ solution of $(S 2)$. The corresponding 
mode shapes are given by the following formulae:

$$
\begin{aligned}
& \tilde{\chi}_{n}^{(1)}(x)=\tanh \left(\frac{K_{n}^{(1)} L}{2}\right) \frac{\sin \left(k_{n}^{(1)} x\right)}{\cos \left(\frac{k_{n}^{(1)} L}{2}\right)}-\tan \left(\frac{k_{n}^{(1)} L}{2}\right) \frac{\sinh \left(K_{n}^{(1)} x\right)}{\cosh \left(\frac{K_{n}^{(1)} L}{2}\right)} \\
& \tilde{\chi}_{n}^{(2)}(x)=K_{n}^{(2)} \tanh \left(\frac{K_{n}^{(2)} L}{2}\right) \frac{\cos \left(k_{n}^{(2)} x\right)}{\cos \left(\frac{k_{n}^{(2)} L}{2}\right)}+k_{n}^{(2)} \tan \left(\frac{k_{n}^{(2)} L}{2}\right) \frac{\cosh \left(K_{n}^{(2)} x\right)}{\cosh \left(\frac{K_{n}^{(2)} L}{2}\right)}
\end{aligned}
$$

Using equation 25, the corresponding expressions for the bulge read:

$$
\begin{aligned}
& \tilde{S}_{n}^{(1)}(x)=-S_{S}\left(k_{n}^{(1)} \tanh \left(\frac{K_{n}^{(1)} L}{2}\right) \frac{\cos \left(k_{n}^{(1)} x\right)}{\cos \left(\frac{k_{n}^{(1)} L}{2}\right)}-K_{n}^{(1)} \tan \left(\frac{k_{n}^{(1)} L}{2}\right) \frac{\cosh \left(K_{n}^{(1)} x\right)}{\cosh \left(\frac{K_{n}^{(1)} L}{2}\right)}\right) \\
& \tilde{S}_{n}^{(2)}(x)=-S_{S} k_{n}^{(2)} K_{n}^{(2)}\left(-\tanh \left(\frac{K_{n}^{(2)} L}{2}\right) \frac{\sin \left(k_{n}^{(2)} x\right)}{\cos \left(\frac{k_{n}^{(2)} L}{2}\right)}+\tan \left(\frac{k_{n}^{(2)} L}{2}\right) \frac{\sinh \left(K_{n}^{(2)} x\right)}{\cosh \left(\frac{K_{n}^{(2)} L}{2}\right)}\right)
\end{aligned}
$$

At this point, one may consider the limit case of a rigid body (distensibility $D \rightarrow 0$ ). According to equation (B.4), for small values of $D, \gamma_{1} \approx \rho D \omega^{2}$ and $\gamma_{2} \approx-\frac{2 \pi}{D T_{S}}$. Thus, according to equation (B.5), $k \approx \pm \sqrt{\rho D} \omega$ and $K \approx \pm \sqrt{\frac{2 \pi}{D T_{S}}}$. By taking these results into account in equation (B.14), one can show that $(\mathrm{S} 1)$ has no non-trivial solution in the limit $D \rightarrow 0$ and that (S2) reduces to the expected equation of motion of a rigid body in surge $\kappa=\frac{\omega^{2} \rho S_{S} L}{-M \omega^{2}+2 K_{a}}=1$.

\section{Orthogonality properties}

Let us verify that the mode shapes are orthogonal. The method follows $[1]$. 
By definition, $\forall x \in\left[-\frac{L}{2}, \frac{L}{2}\right]$, the mode $\tilde{\chi}_{m}$ of type (1) or (2) is solution of:

$$
\frac{1}{\rho D} \frac{\partial^{2} \tilde{\chi}_{m}}{\partial x^{2}}-\frac{1}{\rho D} \frac{1}{K_{0}^{2}} \frac{\partial^{4} \tilde{\chi}_{m}}{\partial x^{4}}=-\omega_{n}^{2} \tilde{\chi}_{m}
$$

Multiplying this equation by mode $\tilde{\chi}_{l}$ (of either type) and integrating over the length of the tube leads to:

$$
\frac{1}{\rho D} \int_{-\frac{L}{2}}^{\frac{L}{2}} \frac{\partial^{2} \tilde{\chi}_{m}}{\partial x^{2}} \tilde{\chi}_{l} d x-\frac{1}{\rho D} \frac{1}{K_{0}^{2}} \int_{-\frac{L}{2}}^{\frac{L}{2}} \frac{\partial^{4} \tilde{\chi}_{m}}{\partial x^{4}} \tilde{\chi}_{l} d x=-\omega_{n}^{2} \int_{-\frac{L}{2}}^{\frac{L}{2}} \tilde{\chi}_{m} \tilde{\chi}_{l} d x
$$

Let us consider the first and second integrals in (B.18). Integrating by parts, one can show:

$$
\begin{aligned}
\int_{-\frac{L}{2}}^{\frac{L}{2}} \frac{\partial^{2} \tilde{\chi}_{m}}{\partial x^{2}} \tilde{\chi}_{l} d x & =\left[\frac{\partial \tilde{\chi}_{m}}{\partial x} \tilde{\chi}_{l}\right]_{-\frac{L}{2}}^{\frac{L}{2}}-\int_{-\frac{L}{2}}^{\frac{L}{2}} \frac{\partial \tilde{\chi}_{m}}{\partial x} \frac{\partial \tilde{\chi}_{l}}{\partial x} d x \\
& =-\int_{-\frac{L}{2}}^{\frac{L}{2}} \frac{\partial \tilde{\chi}_{m}}{\partial x} \frac{\partial \tilde{\chi}_{l}}{\partial x} d x \\
\int_{-\frac{L}{2}}^{\frac{L}{2}} \frac{\partial^{4} \tilde{\chi}_{m}}{\partial x^{4}} \tilde{\chi}_{l} d x & =\left[\frac{\partial^{3} \tilde{\chi}_{m}}{\partial x^{3}} \tilde{\chi}_{l}\right]_{-\frac{L}{2}}^{\frac{L}{2}}-\left[\frac{\partial^{2} \tilde{\chi}_{m}}{\partial x^{2}} \frac{\partial \tilde{\chi}_{l}}{\partial x}\right]_{-\frac{L}{2}}^{\frac{L}{2}}+\int_{-\frac{L}{2}}^{\frac{L}{2}} \frac{\partial^{2} \tilde{\chi}_{m}}{\partial x^{2}} \frac{\partial^{2} \tilde{\chi}_{l}}{\partial x^{2}} d x \\
& =\left.\left(\left.\frac{\partial^{3} \tilde{\chi}_{m}}{\partial x^{3}}\right|_{\frac{L}{2}}-\left.\frac{\partial^{3} \tilde{\chi}_{m}}{\partial x^{3}}\right|_{-\frac{L}{2}}\right) \tilde{\chi}_{l}\right|_{\frac{L}{2}} ^{\frac{L}{2}}+\int_{-\frac{L}{2}}^{\frac{\partial^{2}}{\partial \tilde{\chi}_{m}}} \frac{\partial^{2} \tilde{\chi}_{l}}{\partial x^{2}} d x
\end{aligned}
$$

because the tube ends can not deform $\left(\left.\frac{\partial \tilde{\chi}}{\partial x}\right|_{ \pm \frac{L}{2}}=-\left.\frac{1}{S_{S}} S\right|_{ \pm \frac{L}{2}}=0\right)$ and because $\left.\tilde{\chi}_{l}\right|_{-\frac{L}{2}}=\left.\tilde{\chi}\right|_{\frac{L}{2}}$.

According to the wall equation A.12, at both ends:

$$
\left.p_{i}\right|_{ \pm \frac{L}{2}}=-\left.\frac{1}{D S_{S}} \frac{1}{K_{0}^{2}} \frac{\partial^{2} S}{\partial x^{2}}\right|_{ \pm \frac{L}{2}}=\left.\frac{1}{D} \frac{1}{K_{0}^{2}} \frac{\partial^{3} \chi}{\partial x^{3}}\right|_{ \pm \frac{L}{2}}
$$

Therefore, using the surge equation 18:

$$
M \ddot{X}+K_{a} X=\Delta p_{i} S_{S}=\frac{S_{S}}{D} \frac{1}{K_{0}^{2}}\left(\left.\frac{\partial^{3} \tilde{\chi}}{\partial x^{3}}\right|_{\frac{L}{2}}-\left.\frac{\partial^{3} \tilde{\chi}}{\partial x^{3}}\right|_{-\frac{L}{2}}\right)
$$


Replacing $\tilde{\chi}$ by $\tilde{\chi}_{m}, X$ by $\left.\tilde{\chi}_{m}\right|_{\frac{L}{2}}$ and multiplying by $\left.\tilde{\chi}_{l}\right|_{\frac{L}{2}}$, one obtains:

$$
\left.\left(\left.\frac{\partial^{3} \tilde{\chi}_{m}}{\partial x^{3}}\right|_{\frac{L}{2}}-\left.\frac{\partial^{3} \tilde{\chi}_{m}}{\partial x^{3}}\right|_{-\frac{L}{2}}\right) \tilde{\chi}_{l}\right|_{\frac{L}{2}}=\left.\left.\frac{D K_{0}^{2}}{S_{S}}\left(-M \omega_{m}^{2}+2 K_{a}\right) \tilde{\chi}_{m}\right|_{\frac{L}{2}} \tilde{\chi}_{l}\right|_{\frac{L}{2}}
$$

Thus, in equation B.18:

$$
\begin{aligned}
-\omega_{m}^{2}\left(\int_{-\frac{L}{2}}^{\frac{L}{2}} \tilde{\chi}_{m} \tilde{\chi}_{l} d x+\left.\left.\frac{M}{\rho S_{S}} \tilde{\chi}_{m}\right|_{\frac{L}{2}} \tilde{\chi}_{l}\right|_{\frac{L}{2}}\right)= & -\frac{1}{\rho D} \int_{-\frac{L}{2}}^{\frac{L}{2}} \frac{\partial \tilde{\chi}_{m}}{\partial x} \frac{\partial \tilde{\chi}_{l}}{\partial x} d x-\left.\left.\frac{1}{\rho S_{S}} 2 K_{a} \tilde{\chi}_{m}\right|_{\frac{L}{2}} \tilde{\chi}_{l}\right|_{\frac{L}{2}} \\
& -\frac{1}{\rho D} \frac{1}{K_{0}^{2}} \int_{-\frac{L}{2}}^{\frac{L}{2}} \frac{\partial^{2} \tilde{\chi}_{m}}{\partial x^{2}} \frac{\partial^{2} \tilde{\chi_{l}}}{\partial x^{2}} d x
\end{aligned}
$$

Repeating the same but inverting modes $m$ and $l$, one shows:

$$
-\left(\omega_{m}^{2}-\omega_{l}^{2}\right)\left(\int_{-\frac{L}{2}}^{\frac{L}{2}} \tilde{\chi}_{m} \tilde{\chi}_{l} d x+\left.\left.\frac{M}{\rho S_{S}} \tilde{\chi}_{m}\right|_{\frac{L}{2}} \tilde{\chi}_{l}\right|_{\frac{L}{2}}\right)=0
$$

This last equation proves the modal orthogonality for the scalar product:

$$
\langle f, g\rangle=\int_{-\frac{L}{2}}^{\frac{L}{2}} f(x) g(x) d x+\left.\left.\frac{M}{\rho S_{S}} f\right|_{\frac{L}{2}} g\right|_{\frac{L}{2}}
$$

Let us define the norm $N_{m}$ as:

$$
N_{m}^{2}=\int_{-\frac{L}{2}}^{\frac{L}{2}} \tilde{\chi}_{m}^{2} d x+\left.\frac{M}{\rho S_{S}} \tilde{\chi}_{m}\right|_{\frac{L}{2}} ^{2}
$$

Finally, starting from equation B.18 and adding equation B.21, one can show:

$$
\begin{array}{r}
\frac{1}{\rho D} \int_{-\frac{L}{2}}^{\frac{L}{2}} \frac{\partial^{2} \tilde{\chi}_{m}}{\partial x^{2}} \tilde{\chi}_{l} d x-\frac{1}{\rho D} \frac{1}{K_{0}^{2}} \int_{-\frac{L}{2}}^{\frac{L}{2}} \frac{\partial^{4} \tilde{\chi}_{m}}{\partial x^{4}} \tilde{\chi}_{l} d x-\left.\left.\frac{2 K_{a}}{\rho S_{S}} \tilde{\chi}_{m}\right|_{\frac{L}{2}} \tilde{\chi}_{l}\right|_{\frac{L}{2}} \\
+\left.\frac{1}{\rho D} \frac{1}{K_{0}^{2}}\left(\left.\frac{\partial^{3} \tilde{\chi}_{m}}{\partial x^{3}}\right|_{\frac{L}{2}}-\left.\frac{\partial^{3} \tilde{\chi}_{m}}{\partial x^{3}}\right|_{-\frac{L}{2}}\right) \tilde{\chi}_{l}\right|_{\frac{L}{2}}=-\omega_{n}^{2} N_{n}^{2} \delta_{m l}
\end{array}
$$

in which $\delta_{m l}$ is the Kronecker delta and $N_{m}$ is a norm defined such as $N_{m}^{2}=$ $\int_{-\frac{L}{2}}^{\frac{L}{2}} \tilde{\chi}_{m}^{2} d x+\left.\frac{M}{\rho S_{S}} \tilde{\chi}_{m}\right|_{\frac{L}{2}} ^{2}$. 


\section{References}

[1] P. H. Asl. Design and analysis of micro-electromechanical resonant structures. PhD thesis, The University of Toronto, 2008.

[2] A. Babarit and G. Delhommeau. Theoretical and numerical aspects fo the open source bem solver nemoh. In Proc. of the 11th European Wave and Tidal Energy Conference (EWTEC2015), Nantes, France, 2015.

[3] A. Babarit and P. Ferrant. Investigation on the radiation and diffraction forces of a bulging tube. In Proc. of the International Workshop on Water Waves and Floating Bodies (IWWWFB2011), 2011.

[4] J.R. Chaplin, V. Heller, F.J.M. Farley, G.E. Hearn, and R.C.T. Rainey. Laboratory testing of the anaconda. Philosophical Transactions of the Royal Society A, 370:403-424, 2012.

[5] S. Chiba, M. Waki, R. Kornbluh, and R. Pelrine. Innovative power generators for energy harvesting using electroactive polymer artificial muscles. In Proc. of the SPIE conference 6927 on Electroactive Polymer Actuators and Devices (EAPAD), 2008.

[6] A. F. O. Falcao. Wave energy utilization: a review of technologies. Renewable and Sustainable Energy Reviews, 14:899-918, 2009.

[7] J. Falnes. A resonant point absorber of ocean-wave power. Nature, 256:478-479, 1975.

[8] J. Falnes. A review of wave energy extraction. Marine Structures, $20: 185-201,2007$. 
[9] F.J.M. Farley, R. C. T. Rainey, and J. R. Chaplin. Rubber tubes in the sea. Philosophical Transactions of the Royal Society A, 370:381-402, 2012.

[10] F.J.M. Farley and R.C.T. Rainey. Distensible tube wave energy converter. Patent US20090007557 A1.

[11] L. Formaggia, D. Lamponi, and A. Quarteroni. One-dimensional model for blood flow in arteries. Journal of Engineering Mathematics, 47:251$276,2003$.

[12] K. Gunn and C. Stock-Williams. Quantifying the global wave power resource. Renewable Energy, 44:296-304, 2012.

[13] P. Jean, A. Wattez, G. Ardoise, C. Melis, R. Van Kessel, A. Fourmon, E. Barrabino, J. Heemskerkz, and J. P. Queau. Standing wave tube electro active polymer wave energy converter. In Proc. of the SPIE Smart Structures/NDE conference, 2012.

[14] C.-H. Lee. WAMIT theory manual. Massachussets Institue of Technology. Department of Ocean Engineering., Cambridge, Mass. 02139, 1995.

[15] J.N. Newman. Wave effects on deformable bodies. Applied Ocean Research, 16:47-59, 1994.

[16] J. Pollack and P.F. Jean. Wave energy converter. Patent US20100019498 A1.

[17] A. Quarteroni and L. Formaggia. Mathematical modellaing and numerical simulation of the cardiovascular system. In Handbook of Numeri- 
cal Analysis, volume 12 of Computational Models for the Human Body, pages 3-127. 2004.

[18] O. H. Yeoh. Some forms of the strain energy function for rubber. Rubber chemistry and technology, 66:754-771, 1993. 